ESAIM: COCV 20 (2014) 416-441

DOI: $10.1051 / \mathrm{cocv} / 2013069$
ESAIM: Control, Optimisation and Calculus of Variations

www.esaim-cocv.org

\title{
A CERTIFIED REDUCED BASIS METHOD FOR PARAMETRIZED ELLIPTIC OPTIMAL CONTROL PROBLEMS*
}

\author{
Mark KärCher ${ }^{1}$ AND MARTin A. GrepL ${ }^{2}$
}

\begin{abstract}
In this paper, we employ the reduced basis method as a surrogate model for the solution of linear-quadratic optimal control problems governed by parametrized elliptic partial differential equations. We present a posteriori error estimation and dual procedures that provide rigorous bounds for the error in several quantities of interest: the optimal control, the cost functional, and general linear output functionals of the control, state, and adjoint variables. We show that, based on the assumption of affine parameter dependence, the reduced order optimal control problem and the proposed bounds can be efficiently evaluated in an offline-online computational procedure. Numerical results are presented to confirm the validity of our approach.
\end{abstract}

Mathematics Subject Classification. 49K20, 49M29, 35J25, 65N15, 65K05, 93C20.

Received May 25, 2012. Revised April 24, 2013.

Published online March 7, 2014.

\section{INTRODUCTION}

Many problems in science and engineering can be modeled in terms of optimal control problems governed by parametrized partial differential equations (PDEs). While the PDE describes the underlying system or component behavior, the parameters often serve to identify a particular configuration of the component - such as boundary and initial conditions, material properties, and geometry. Parameter-dependent PDE-constrained optimal control problems appear in a wide range of applications, e.g., ranging from pollution control problems $[6,21,27]$, to Stokes flow [21], to (Helmholtz) acoustics [30], and to the optimal design of bypass grafts [26]. The solution of these problems using classical discretization techniques such as finite elements or finite volumes is computationally expensive and time-consuming. One way to decrease the computational burden is the surrogate model approach, where the original high-dimensional model is replaced by its reduced order approximation. These ideas have received a lot of attention in the past and various model order reduction techniques have been used in this context. We refer, e.g., to $[1,17,18,30,31]$ for proper orthogonal decomposition (POD), to [12] for

Keywords and phrases. Optimal control, reduced basis method, a posteriori error estimation, model order reduction, parameterdependent systems.

* This work was supported by the Excellence Initiative of the German federal and state governments and the German Research Foundation through Grant GSC 111.

1 Aachen Institute for Advanced Study in Computational Engineering Science (AICES), RWTH Aachen University, Schinkelstraße 2, 52062 Aachen, Germany. kaercher@aices.rwth-aachen.de

2 Numerical Mathematics, RWTH Aachen University, Templergraben 55, 52056 Aachen, Germany. grepl@igpm.rwth-aachen.de 
reduction based on inertial manifolds, and to $[4,6,13-15,21,26,27,30]$ for reduced basis methods. However, the solution of the reduced order optimal control problem is generally suboptimal and reliable error estimation is thus crucial.

A posteriori error bounds have been proposed previously for POD approximations in [31] to estimate the distance between the computed suboptimal control and the unknown optimal control; also see [30,35] for an application of this approach to other model order reduction techniques. In $[4,6]$, reduced basis approximations and associated a posteriori error estimation procedures have been derived to estimate the error in the optimal value of the cost functional. However, the bounds in [31], although rigorous, require solution of the underlying high-dimensional problem and are thus online-inefficient; whereas the estimates in $[4,6]$, although efficient to evaluate, are not rigorous upper bounds for the error.

In this paper we employ the reduced basis method as a surrogate model for the solution of the optimal control problem. The reduced basis method is a model order reduction technique which provides efficient yet reliable approximations to solutions of parametrized partial differential equations in the many-query or realtime context [20,25]; also see [29] for a review of contributions to the methodology and further references. The methodology is thus ideally suited for the solution of parametrized PDE-constrained optimal control problems. In a recent note [8] we considered elliptic optimal control problems involving a scalar control and proposed rigorous and efficiently evaluable a posteriori error bounds for the optimal control and the associated cost functional. Our approach thus allows not only the efficient real-time solution of the reduced optimal control problem, but also the efficient real-time evaluation of the quality of the suboptimal solution. We also refer to [16] for an extension of these ideas to problems involving distributed controls. Recently, a reduced basis approach to optimal control problems based on a saddle-point formulation has been considered in [21].

Here, we extend the work in [8] in the following directions: First, we introduce new a posteriori error bounds based on a dual approach. To this end, we note that each component of the optimal control is in fact a linear output of the optimality system. We thus build on the ideas in [24] to obtain superconvergent a posteriori error bounds for the optimal control and, more generally, for linear output functionals of the state, adjoint, and control. We show that the duality-based error bounds can be efficiently evaluated using the standard offlineonline computational procedure. Recall that dual techniques have been used successfully in $[5,10,25,32]$ to improve the convergence of reduced basis output approximations and the associated output bounds. We also generalize the optimal control problem discussed in [8] by (i) considering problems involving multiple control inputs; and (ii) introducing a more general parametrization of the cost functional. Concerning the latter, we show that our approach directly applies to cost functionals involving an (affinely) parametrized desired state (or control) and that we can also allow the regularization parameter to vary.

This paper is organized as follows. In Section 2 we briefly review the reduced basis recipe for linear secondorder elliptic partial differential equations (PDEs). The optimal control problem is discussed in Section 3: we start with the general problem statement, state the first order optimality conditions, and illustrate how the reduced basis method can be applied as a surrogate model. In Section 4 we introduce rigorous and efficiently evaluable a posteriori error bounds for several quantities of interest: the optimal control, the associated cost functional, and general linear output functionals of the state, adjoint and control variables. Finally, in Section 5, we present numerical results for a stationary heat conduction model problem that show the validity of our approach.

\section{THE REDUCED BASIS METHOD}

We start with the general problem formulation and a brief review of the reduced basis approximation and associated a posteriori error estimation procedures for linear second-order elliptic partial differential equations. The derivation of our a posteriori error bounds for the optimal control problem in Section 4 relies heavily on these - by now - standard results. 


\subsection{Problem formulation}

Let $Y_{\mathrm{e}}$ with $\mathrm{H}_{0}^{1}(\Omega) \subset Y_{\mathrm{e}} \subset \mathrm{H}^{1}(\Omega)$ be a Hilbert space over the bounded Lipschitz domain $\Omega \subset \mathbb{R}^{d}, d=1,2,3$, with boundary $\Gamma .^{3}$ The inner product and induced norm associated with $Y_{\mathrm{e}}$ are given by $(\cdot, \cdot)_{Y_{\mathrm{e}}}$ and $\|\cdot\|_{Y_{\mathrm{e}}}=$ $\sqrt{(\cdot, \cdot)_{Y_{\mathrm{e}}}}$, respectively; we assume that the norm $\|\cdot\|_{Y_{\mathrm{e}}}$ is equivalent to the $\mathrm{H}^{1}(\Omega)$-norm [28]. In anticipation of the optimal control problem considered in Section 3 we also introduce the finite-dimensional control space $\mathcal{U}=\mathbb{R}^{m}, m \in \mathbb{N}$, together with its standard euclidean inner product $(\cdot, \cdot)_{\mathcal{U}}$ and induced norm $\|\cdot\|_{\mathcal{U}}=\sqrt{(\cdot, \cdot)_{\mathcal{U}}}$. We denote the associated dual spaces of $Y_{\mathrm{e}}$ and $\mathcal{U}$ by $Y_{\mathrm{e}}^{\prime}$ and $\mathcal{U}^{\prime}$, respectively. Furthermore, we let $\mathcal{D} \subset \mathbb{R}^{P}$ be a prescribed $P$-dimensional compact parameter set in which our $P$-tuple (input) parameter $\mu=\left(\mu_{1}, \ldots, \mu_{P}\right)$ resides.

We next introduce the parameter-dependent bilinear form $a(\cdot, \cdot ; \mu): Y_{\mathrm{e}} \times Y_{\mathrm{e}} \rightarrow \mathbb{R}$. We shall assume that $a(\cdot, \cdot ; \mu)$ is continuous,

$$
0<\gamma_{\mathrm{e}}(\mu)=\sup _{w \in Y_{\mathrm{e}} \backslash\{0\}} \sup _{v \in Y_{\mathrm{e}} \backslash\{0\}} \frac{a(w, v ; \mu)}{\|w\|_{Y_{\mathrm{e}}}\|v\|_{Y_{\mathrm{e}}}} \leq \gamma_{0}<\infty, \quad \forall \mu \in \mathcal{D}
$$

coercive,

$$
\alpha_{\mathrm{e}}(\mu)=\inf _{v \in Y_{\mathrm{e}} \backslash\{0\}} \frac{a(v, v ; \mu)}{\|v\|_{Y_{\mathrm{e}}}^{2}} \geq \alpha_{0}>0, \quad \forall \mu \in \mathcal{D},
$$

and satisfies the affine parameter dependence

$$
a(w, v ; \mu)=\sum_{q=1}^{Q_{a}} \Theta_{a}^{q}(\mu) a^{q}(w, v), \quad \forall w, v \in Y_{\mathrm{e}}, \quad \forall \mu \in \mathcal{D},
$$

for some (preferably) small integer $Q_{a}$. Here, the coefficient functions $\Theta_{a}^{q}(\cdot): \mathcal{D} \rightarrow \mathbb{R}$ are continuous and depend on $\mu$, but the continuous bilinear forms $a^{q}(\cdot, \cdot): Y_{\mathrm{e}} \times Y_{\mathrm{e}} \rightarrow \mathbb{R}$ do not depend on $\mu$. We recall that the affine parameter dependence is crucial for the efficiency of the reduced basis method (see [9] for an extension to nonaffine problems). Finally, we introduce the continuous and linear operator $\mathcal{B}: \mathcal{U} \rightarrow Y_{\mathrm{e}}^{\prime}$, given by

$$
\mathcal{B} u=\sum_{i=1}^{m} b_{i}(\cdot) u_{i}
$$

where $b_{i}(\cdot): Y_{\mathrm{e}} \rightarrow \mathbb{R}, 1 \leq i \leq m$, are given bounded linear functionals and $u=\left(u_{1}, \ldots, u_{m}\right)^{T} \in \mathcal{U}$ is the control with control components $u_{i} \in \mathbb{R}, 1 \leq i \leq m$. For simplicity, we assume that the functionals $b_{1}, \ldots, b_{m}$ do not depend on the parameter; however, (affine) parameter dependence of the $b_{i}$ and thus of the operator $\mathcal{B}$ itself is readily admitted [29].

We may now introduce the exact (or analytic) problem statement: For any parameter $\mu \in \mathcal{D}$ and fixed control $u \in \mathcal{U}$, find $y_{\mathrm{e}} \equiv y_{\mathrm{e}}(\mu) \in Y_{\mathrm{e}}^{4}$ such that

$$
a\left(y_{\mathrm{e}}, v ; \mu\right)=\langle\mathcal{B} u, v\rangle_{Y_{\mathrm{e}}^{\prime}, Y_{\mathrm{e}}}, \quad \forall v \in Y_{\mathrm{e}},
$$

where $\langle\cdot, \cdot\rangle_{Y_{\mathrm{e}}^{\prime}, Y_{\mathrm{e}}}$ denotes the dual pairing between $Y_{\mathrm{e}}^{\prime}$ and $Y_{\mathrm{e}}$. This equation will act as the PDE constraint for the optimal control problem considered in Section 3.

\subsubsection{Truth approximation}

We next introduce a "truth" finite element approximation space $Y \subset Y_{\mathrm{e}}$ of very large dimension $\mathcal{N}$. Note that $Y$ shall inherit the inner product and norm from $Y_{\mathrm{e}}:(\cdot, \cdot)_{Y}=(\cdot, \cdot)_{Y_{\mathrm{e}}}$ and $\|\cdot\|_{Y}=\|\cdot\|_{Y_{e}}$. Clearly, the

\footnotetext{
${ }^{3}$ The subscript e denotes "exact".

${ }^{4}$ Here and in the following we often drop the dependence on $\mu$ to simplify notation.
} 
continuity and coercivity properties of the bilinear form $a$ are inherited by the "truth" approximation, i.e.,

$$
\gamma(\mu)=\sup _{w \in Y \backslash\{0\}} \sup _{v \in Y \backslash\{0\}} \frac{a(w, v ; \mu)}{\|w\|_{Y}\|v\|_{Y}} \leq \gamma_{\mathrm{e}}(\mu) \leq \gamma_{0}<\infty, \quad \forall \mu \in \mathcal{D},
$$

and

$$
\alpha(\mu)=\inf _{v \in Y \backslash\{0\}} \frac{a(v, v ; \mu)}{\|v\|_{Y}^{2}} \geq \alpha_{\mathrm{e}}(\mu) \geq \alpha_{0}>0, \quad \forall \mu \in \mathcal{D} .
$$

Our truth approximation to the exact problem (2.5) is then: Given $\mu \in \mathcal{D}$ and $u \in \mathcal{U}$, find $y \equiv y(\mu) \in Y$ such that

$$
a(y, v ; \mu)=\langle\mathcal{B} u, v\rangle_{Y^{\prime}, Y}, \quad \forall v \in Y .
$$

We shall assume that the truth space $Y$ is sufficiently rich such that $y(\mu)$ and $y_{\mathrm{e}}(\mu)$ are indistinguishable. We further recall that the reduced basis approximation shall be built upon - and the reduced basis error thus evaluated with respect to - the truth solution $y(\mu) \in Y$.

\subsection{Reduced basis approximation}

We assume that we are given the nested parameter samples $\mathcal{D}_{N} \equiv\left\{\mu^{1} \in \mathcal{D}, \ldots, \mu^{N} \in \mathcal{D}\right\}, 1 \leq N \leq N_{\max }$, and associated Lagrangian reduced basis spaces, $Y_{N}=\operatorname{span}\left\{y\left(\mu^{n}\right), 1 \leq n \leq N\right\}, 1 \leq N \leq N_{\max }$, consisting of so-called snapshots, i.e., solutions $y$ of (2.8) for all $\mu \in \mathcal{D}_{N}$. In actual practice, we construct $\mathcal{D}_{N}$ using a Greedy sampling procedure [33]. For stability considerations the snapshots are orthogonalized to obtain a set

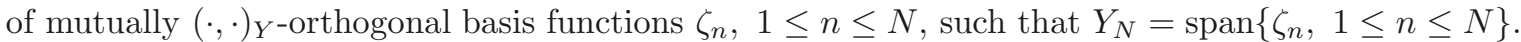

The reduced basis approximation is then obtained by a standard Galerkin projection onto the reduced basis space: Given $\mu \in \mathcal{D}$ and $u \in \mathcal{U}$, find $y_{N} \equiv y_{N}(\mu) \in Y_{N}$ such that

$$
a\left(y_{N}, v ; \mu\right)=\langle\mathcal{B} u, v\rangle_{Y^{\prime}, Y}, \quad \forall v \in Y_{N} .
$$

\subsection{A posteriori error estimation}

A posteriori error estimation procedures play a decisive role in the reduced basis method. The error bounds help to (i) efficiently and rigorously assess the error introduced by the reduced basis approximation; and (ii) drive the Greedy procedure for generating the reduced basis space $Y_{N}$.

To begin, we specify the inner product $(v, w)_{Y}=\frac{1}{2}\left(a\left(v, w ; \mu^{\text {ref }}\right)+a\left(w, v ; \mu^{\text {ref }}\right)\right)$, where $\mu^{\text {ref }} \in \mathcal{D}$ is a reference parameter value (for $a(\cdot, \cdot ; \mu)$ symmetric we then have $(v, w)_{Y}=a\left(v, w ; \mu^{\text {ref }}\right)$ ). We next assume that we are given a positive lower bound $\alpha_{\mathrm{LB}}(\cdot): \mathcal{D} \rightarrow \mathbb{R}_{+}$for the coercivity constant $\alpha(\mu)$ such that

$$
\alpha(\mu) \geq \alpha_{\mathrm{LB}}(\mu) \geq \alpha_{0}>0, \quad \forall \mu \in \mathcal{D} ;
$$

various recipes exist to construct this lower bound $[11,25,34]$. We can now state the standard $Y$-norm error bound in Theorem 2.1. We repeat the short proof here since the derivation of our new error bounds in the optimal control setting is, in fact, an extension of this standard result.

Theorem 2.1. Let $y \in Y$ be the solution to the truth problem (2.8) and $y_{N} \in Y_{N}$ be the corresponding reduced basis approximation satisfying (2.9). The error then satisfies

$$
\left\|y-y_{N}\right\|_{Y} \leq \Delta_{N}(\mu) \equiv \frac{\|r(\cdot ; \mu)\|_{Y^{\prime}}}{\alpha_{\mathrm{LB}}(\mu)}, \quad \forall \mu \in \mathcal{D},
$$

where the residual $r(\cdot ; \mu): Y \rightarrow \mathbb{R}$ is given by $r(v ; \mu)=\langle\mathcal{B} u, v\rangle_{Y^{\prime}, Y}-a\left(y_{N}, v ; \mu\right), \forall v \in Y$, and its dual norm by $\|r(\cdot ; \mu)\|_{Y^{\prime}} \equiv \sup _{v \in Y \backslash\{0\}} r(v ; \mu) /\|v\|_{Y}$. 
Proof. It follows from (2.8) and the definition of the residual $r(v ; \mu)$ that the reduced basis error, $y-y_{N}$, satisfies

$$
a\left(y-y_{N}, v ; \mu\right)=\langle\mathcal{B} u, v\rangle_{Y^{\prime}, Y}-a\left(y_{N}, v ; \mu\right)=r(v ; \mu), \quad \forall v \in Y .
$$

Choosing $v=y-y_{N}$ in (2.12), invoking (2.7), (2.10), and the dual norm of the residual, we obtain

$$
\alpha_{\mathrm{LB}}(\mu)\left\|y-y_{N}\right\|_{Y}^{2} \leq a\left(y-y_{N}, y-y_{N} ; \mu\right) \leq\|r(\cdot ; \mu)\|_{Y^{\prime}}\left\|y-y_{N}\right\|_{Y},
$$

from which the result directly follows.

\subsection{Computational procedure}

The affine parameter dependence of the bilinear form $a$ allows an efficient offline-online computational procedure to evaluate the reduced basis approximation $y_{N}$ and associated a posteriori error bound $\Delta_{N}(\mu)$. We briefly recall the essential steps of the former and then summarize the online computational cost to evaluate $y_{N}$ and $\Delta_{N}(\mu)$; we refer to [29] for more details.

We express $y_{N}(\mu)=\sum_{i=1}^{N} y_{N i}(\mu) \zeta_{i}$ and choose as test functions $v=\zeta_{i}, 1 \leq i \leq N$, in (2.9). It then follows that $\underline{y}_{N}(\mu)=\left[y_{N 1}(\mu), \ldots, y_{N N}(\mu)\right]^{T} \in \mathbb{R}^{N}$ satisfies

$$
A_{N}(\mu) \underline{y}_{N}(\mu)=B_{N} u
$$

where $A_{N}(\mu) \in \mathbb{R}^{N \times N}$ and $B_{N}(\mu) \in \mathbb{R}^{N \times m}$ are matrices with entries $\left(A_{N}(\mu)\right)_{i j}=a\left(\zeta_{j}, \zeta_{i} ; \mu\right), 1 \leq i, j \leq N$, and $\left(B_{N}\right)_{i j}=b_{j}\left(\zeta_{i}\right), 1 \leq i \leq N, 1 \leq j \leq m$, respectively. Invoking the affine parameter dependence (2.3) yields the expansion $A_{N}(\mu)=\sum_{q=1}^{\overline{Q_{a}}} \Theta_{a}^{q}(\mu) A_{N}^{q}$, where the parameter-independent matrices $A_{N}^{q} \in \mathbb{R}^{N \times N}$ are given by $\left(A_{N}^{q}\right)_{i j}=a^{q}\left(\zeta_{j}, \zeta_{i}\right), 1 \leq i, j \leq N, 1 \leq q \leq Q_{a}$.

The offline computational cost clearly depends on $\mathcal{N}$ : we need to solve for the basis functions $\zeta_{i}, 1 \leq i \leq N$, and precompute the parameter-independent matrices $A_{N}^{q}, 1 \leq q \leq Q_{a}$, and $B_{N}$; the offline cost associated to $\Delta_{N}(\mu)$ also depends on $\mathcal{N}$ [29]. In the online stage, for each new parameter value $\mu$, we assemble the parameterdependent matrix $A_{N}(\mu)$ at cost $\mathcal{O}\left(Q_{a} N^{2}\right)$ and then solve $(2.14)$ at cost $\mathcal{O}\left(N^{3}\right)$. We subsequently evaluate $\Delta_{N}(\mu)$ online at cost $\mathcal{O}\left(\left(Q_{a} N+m\right)^{2}\right)$. The computational cost in the online stage is thus independent of the truth finite element dimension $\mathcal{N}$.

\section{Parametrized optimal Control problem}

In this section we introduce the parametrized linear-quadratic optimal control problem with elliptic PDE constraint. We recall the first-order necessary (and in our case sufficient) optimality conditions and employ the reduced basis method for the efficient solution of the resulting optimality system.

\subsection{General problem statement}

We consider the problem setting as stated in Section 2. We next introduce the quadratic cost functional $J(\cdot, \cdot ; \mu): Y_{\mathrm{e}} \times \mathcal{U} \rightarrow \mathbb{R}$ given by

$$
J(y, u ; \mu)=\frac{1}{2}\left\|y-y_{d}(\mu)\right\|_{L^{2}(D)}^{2}+\frac{\lambda}{2}\left\|u-u_{d}\right\|_{\mathcal{U}}^{2},
$$

where $D \subset \Omega$ or $D \subset \Gamma$ is a measurable set; $y_{d}(\mu) \in \mathrm{L}^{2}(D), \forall \mu \in \mathcal{D}$, and $u_{d} \in \mathcal{U}=\mathbb{R}^{m}$ are the desired state and control, respectively; and $\lambda>0$ is the regularization parameter. We shall assume that $y_{d}(\mu)$ satisfies an affine parametric dependence, i.e., we can express

$$
y_{d}(\mu)=\sum_{q=1}^{Q_{y d}} \Theta_{y d}^{q}(\mu) y_{d}^{q}(x), \quad \forall \mu \in \mathcal{D},
$$


for some (preferably) small integer $Q_{y d}$. We note that, similar to the affine decomposition (2.3), the coefficient functions $\Theta_{y d}^{q}(\cdot): \mathcal{D} \rightarrow \mathbb{R}$ are continuous and depend on $\mu$, but the functions $y_{d}^{q} \in \mathrm{L}^{2}(D)$ do not depend on $\mu$. For simplicity, we assume that the desired control $u_{d}$ is parameter-independent; however, (affine) parameter dependence is readily admitted. Furthermore, for our model problem in Section 5 we will also consider the regularization parameter $\lambda$ itself as a varying input parameter.

We can now state the parametrized optimal control problem as

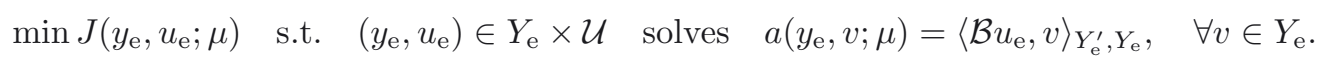

It follows from our assumptions that there exists a unique optimal solution $\left(y_{\mathrm{e}}^{*}, u_{\mathrm{e}}^{*}\right)$ to $\left(\mathrm{P}_{\mathrm{e}}\right)[19]$. Employing a Lagrangian approach we obtain the first-order optimality system consisting of the state equation, the adjoint equation, and the optimality condition: Given $\mu \in \mathcal{D}$, the optimal solution $\left(y_{\mathrm{e}}^{*}, p_{\mathrm{e}}^{*}, u_{\mathrm{e}}^{*}\right) \in Y_{\mathrm{e}} \times Y_{\mathrm{e}} \times \mathcal{U}$ satisfies $^{5}$

$$
\begin{aligned}
a\left(y_{\mathrm{e}}^{*}, \phi ; \mu\right) & =\left\langle\mathcal{B} u_{\mathrm{e}}^{*}, \phi\right\rangle_{Y_{\mathrm{e}}^{\prime}, Y_{\mathrm{e}},} & & \forall \phi \in Y_{\mathrm{e}}, \\
a\left(\varphi, p_{\mathrm{e}}^{*} ; \mu\right) & =\left(y_{d}(\mu)-y_{\mathrm{e}}^{*}, \varphi\right)_{\mathrm{L}^{2}(D)}, & & \forall \varphi \in Y_{\mathrm{e}}, \\
\left(\lambda\left(u_{\mathrm{e}}^{*}-u_{d}\right)-\mathcal{B}^{\star} p_{\mathrm{e}}^{*}, \psi\right)_{\mathcal{U}} & =0, & & \forall \psi \in \mathcal{U} .
\end{aligned}
$$

Here, $p_{\mathrm{e}}$ is the adjoint variable and the superscript ${ }^{*}$ denotes optimality. Furthermore, the linear and bounded dual operator of $\mathcal{B}$ in $(3.3 \mathrm{c})$ is given by $\mathcal{B}^{\star}: Y_{\mathrm{e}} \rightarrow \mathcal{U}$, where we identify $\left(Y_{\mathrm{e}}^{\prime}\right)^{\prime}$ with $Y_{\mathrm{e}}$ and $\mathcal{U}^{\prime}$ with $\mathcal{U}$. From the relationship

$$
\langle\mathcal{B} u, \phi\rangle_{Y_{\mathrm{e}}^{\prime}, Y_{\mathrm{e}}}=\sum_{i=1}^{m} b_{i}(\phi) u_{i}=\left(\mathcal{B}^{\star} \phi, u\right)_{\mathcal{U}},
$$

it follows that, for given $\phi \in Y_{\mathrm{e}}$, the dual operator $\mathcal{B}^{\star} \phi$ can be expressed as

$$
\mathcal{B}^{\star} \phi=\left(b_{1}(\phi), \ldots, b_{m}(\phi)\right)^{T} .
$$

We note that for the linear-quadratic optimal control problem $\left(\mathrm{P}_{\mathrm{e}}\right)$ the first-order conditions (3.3) are necessary and sufficient for the optimality of $\left(y_{\mathrm{e}}^{*}, u_{\mathrm{e}}^{*}\right)$.

\subsection{Truth approximation}

In general, we of course cannot expect to find an analytic solution to (3.3). We thus replace the infinitedimensional Ansatz-space $Y_{\mathrm{e}}$ for the PDE constraint by our truth approximation space $Y \subset Y_{\mathrm{e}}$ introduced in Section 2.1.1. We also note that we do not discretize the control space $\mathcal{U}$, i.e., we have $\mathcal{U}=\mathbb{R}^{m}$, since we assume the number of control inputs $m$ to be moderate. The corresponding truth optimal control problem is then given by

$$
\min J(y, u ; \mu) \quad \text { s.t. } \quad(y, u) \in Y \times \mathcal{U} \quad \text { solves } a(y, v ; \mu)=\langle\mathcal{B} u, v\rangle_{Y^{\prime}, Y}, \quad \forall v \in Y .
$$

The associated first-order optimality system reads: Given $\mu \in \mathcal{D}$, the optimal solution $\left(y^{*}, p^{*}, u^{*}\right) \in Y \times Y \times \mathcal{U}$ satisfies

$$
\begin{aligned}
a\left(y^{*}, \phi ; \mu\right) & =\left\langle\mathcal{B} u^{*}, \phi\right\rangle_{Y^{\prime}, Y}, & & \forall \phi \in Y, \\
a\left(\varphi, p^{*} ; \mu\right) & =\left(y_{d}(\mu)-y^{*}, \varphi\right)_{\mathrm{L}^{2}(D)}, & & \forall \varphi \in Y, \\
\left(\lambda\left(u^{*}-u_{d}\right)-\mathcal{B}^{\star} p^{*}, \psi\right)_{\mathcal{U}} & =0, & & \forall \psi \in \mathcal{U} .
\end{aligned}
$$

We note that the Ansatz and test spaces are identical for the state and adjoint equations. This corresponds to a "first-discretize-then-optimize" approach and ensures that the solution of the optimality system (3.6) is indeed also an optimal solution of the truth optimal control problem (P).

The optimality system (3.6) constitutes a coupled set of equations of dimension $2 \mathcal{N}+m$ and is thus expensive to solve, especially if one is interested in various values of $\mu \in \mathcal{D}$. Our goal is therefore to significantly speed up the solution of (3.6) by employing the reduced basis approximation as a surrogate model for the PDE constraint in $(\mathrm{P})$.

\footnotetext{
${ }^{5}$ We again note that we drop the dependence on $\mu$ to simplify notation, i.e., we write $y_{\mathrm{e}}=y_{\mathrm{e}}(\mu), p_{\mathrm{e}}=p_{\mathrm{e}}(\mu)$, and $u_{\mathrm{e}}=u_{\mathrm{e}}(\mu)$.
} 


\subsection{Reduced basis approximation}

We first assume that we are given the "integrated" reduced basis spaces

$$
Y_{N}=\operatorname{span}\left\{\zeta_{n}, 1 \leq n \leq N\right\}=\operatorname{span}\left\{y^{*}\left(\mu^{n}\right), p^{*}\left(\mu^{n}\right), 1 \leq n \leq N / 2\right\}, \quad 1 \leq N / 2 \leq N_{\max } / 2,
$$

where the $\zeta_{n}, 1 \leq n \leq N$, are mutually $(\cdot, \cdot)_{Y}$-orthogonal basis functions and $N, N_{\max }$ are even. Note that we integrate both state and adjoint snapshots in $Y_{N}$; thus the term "integrated." We discuss the reason for doing so below. We comment on the Greedy sampling procedure to construct the spaces $Y_{N}$ in Section 4.5.

We next replace the truth approximation of the PDE constraint in $(\mathrm{P})$ by its reduced basis approximation. The reduced basis optimal control problem is thus given by

$$
\min J\left(y_{N}, u_{N} ; \mu\right) \quad \text { s.t. } \quad\left(y_{N}, u_{N}\right) \in Y_{N} \times \mathcal{U} \quad \text { solves } a\left(y_{N}, v ; \mu\right)=\left\langle\mathcal{B} u_{N}, v\right\rangle_{Y^{\prime}, Y}, \quad \forall v \in Y_{N} .
$$

We can also directly state the associated first-order optimality system: Given $\mu \in \mathcal{D}$, find $\left(y_{N}^{*}, p_{N}^{*}, u_{N}^{*}\right) \in$ $Y_{N} \times Y_{N} \times \mathcal{U}$ such that

$$
\begin{aligned}
a\left(y_{N}^{*}, \phi ; \mu\right) & =\left\langle\mathcal{B} u_{N}^{*}, \phi\right\rangle_{Y^{\prime}, Y}, & & \forall \phi \in Y_{N}, \\
a\left(\varphi, p_{N}^{*} ; \mu\right) & =\left(y_{d}(\mu)-y_{N}^{*}, \varphi\right)_{\mathrm{L}^{2}(D)}, & & \forall \varphi \in Y_{N}, \\
\left(\lambda\left(u_{N}^{*}-u_{d}\right)-\mathcal{B}^{\star} p_{N}^{*}, \psi\right)_{\mathcal{U}} & =0, & & \forall \psi \in \mathcal{U} .
\end{aligned}
$$

The reduced basis optimality system is only of dimension $2 N+m$ and can be evaluated efficiently using an offline-online computational decomposition. Before presenting the details in the next section we make several remarks.

We note that we use a single reduced basis Ansatz and test space for the state and adjoint equations. The reason is twofold: first, the reduced basis optimality system (3.8) reflects the reduced basis optimal control problem $\left(\mathrm{P}_{\mathrm{N}}\right)$ only if the spaces of the state and adjoint equations are identical; and second, using different spaces may result in an unstable system (3.8). This issue is closely related to the stability of reduced basis formulations for saddle point problems, see [7] for details. If we use the same space $Y_{N}$ for the state and the adjoint equation, on the other hand, the system (3.8) is provably stable. Finally, since the state and adjoint solutions need to be well-approximated using the single space $Y_{N}$, we choose "integrated" spaces, i.e., we integrate both snapshots of the state and adjoint equations into the space $Y_{N}$ defined in (3.7).

\subsection{Computational procedure}

We now turn to the computational details of the reduced basis approximation of the optimality system. To this end, we express the reduced basis state and adjoint solutions as $y_{N}(\mu)=\sum_{i=1}^{N} y_{N i}(\mu) \zeta_{i}$ and $p_{N}(\mu)=\sum_{i=1}^{N} p_{N i}(\mu) \zeta_{i}$ and denote the coefficient vectors by $\underline{y}_{N}(\mu)=\left[y_{N 1}(\mu), \ldots, y_{N N}(\mu)\right]^{T} \in \mathbb{R}^{N}$ and $\underline{p}_{N}(\mu)=\left[p_{N 1}(\mu), \ldots, p_{N N}(\mu)\right]^{T} \in \mathbb{R}^{N}$, respectively. If we choose as test functions $\phi=\zeta_{i}, 1 \leq i \leq N$, $\varphi=\zeta_{i}, 1 \leq i \leq N$, and $\psi=e_{i}, 1 \leq i \leq m$, in (3.8), where $e_{i}$ denotes the $i$-th unit vector in $\mathbb{R}^{m}$, the reduced basis optimality system can be expressed in terms of the $(2 N+m) \times(2 N+m)$ linear system

$$
\left[\begin{array}{ccc}
A_{N}(\mu) & 0 & -B_{N} \\
D_{N} & A_{N}(\mu)^{T} & 0 \\
0 & -B_{N}^{T} & \lambda I_{m}
\end{array}\right]\left[\begin{array}{c}
\underline{y}_{N} \\
\underline{p}_{N} \\
u_{N}
\end{array}\right]=\left[\begin{array}{c}
0 \\
Y_{d, N}(\mu) \\
\lambda u_{d}
\end{array}\right] .
$$

Here, $A_{N}(\mu) \in \mathbb{R}^{N \times N}$ and $B_{N} \in \mathbb{R}^{N \times m}$ are the matrices defined in Section 2.4; $D_{N} \in \mathbb{R}^{N \times N}$ is a matrix with the entries $\left(D_{N}\right)_{i j}=\left(\zeta_{j}, \zeta_{i}\right)_{\mathrm{L}^{2}(D)}, 1 \leq i, j \leq N ; Y_{d, N}(\mu) \in \mathbb{R}^{N}$ is a vector with entries $\left(Y_{d, N}\right)_{i}=$ $\left(y_{d}(\mu), \zeta_{i}\right)_{\mathrm{L}^{2}(D)}, 1 \leq i \leq N$; and $I_{m} \in \mathbb{R}^{m \times m}$ denotes the identity matrix. Invoking the affine parameter dependence (2.3) yields the expansion $A_{N}(\mu)=\sum_{q=1}^{Q_{a}} \Theta_{a}^{q}(\mu) A_{N}^{q}$ as discussed in Section 2.4. Similarly, due to the affine parameter dependence (3.2) of the desired state, we obtain $Y_{d, N}(\mu)=\sum_{q=1}^{Q_{y d}} \Theta_{y d}^{q}(\mu) Y_{d, N}^{q}$, where the 
parameter-independent vectors $Y_{d, N}^{q} \in \mathbb{R}^{N}$ are given by $\left(Y_{d, N}^{q}\right)_{i}=\left(y_{d}^{q}, \zeta_{i}\right)_{\mathrm{L}^{2}(D)}, 1 \leq i \leq N, 1 \leq q \leq Q_{y d}$. Finally, to allow an efficient evaluation of the cost functional in the online stage, we also assemble and store the matrix $\tilde{Y}_{d} \in \mathbb{R}^{Q_{y d} \times Q_{y d}}$ given by $\left(\tilde{Y}_{d}\right)_{p q}=\left(y_{d}^{p}, y_{d}^{q}\right)_{\mathrm{L}^{2}(D)}, 1 \leq p, q \leq Q_{y d}$.

The offline-online decomposition is now clear. In the offline stage - performed only once - we first construct the reduced basis space $Y_{N}$. We then assemble the parameter-independent quantities $A_{N}^{q}, 1 \leq q \leq Q_{a}, D_{N}, B_{N}$, $Y_{d, N}^{q}, 1 \leq q \leq Q_{y d}$, and $\tilde{Y}_{d}$. The computational cost clearly depends on the truth finite element dimension $\mathcal{N}$. In the online stage - for each new parameter value $\mu$ - we first assemble the parameter-dependent quantities $A_{N}(\mu)$ and $Y_{d, N}(\mu)$ in $\mathcal{O}\left(Q_{a} N^{2}\right)$ and $\mathcal{O}\left(Q_{y d} N\right)$ operations, respectively. We then solve the reduced basis optimality system $(3.9)$ at $\operatorname{cost} \mathcal{O}\left((2 N+m)^{3}\right)$. Given the reduced basis optimal solution, the cost functional can then be evaluated efficiently from

$$
J\left(y_{N}, u_{N}\right)=\frac{1}{2} \underline{y}_{N}^{T} D_{N} \underline{y}_{N}-\sum_{q=1}^{Q_{y d}} \Theta_{y d}^{q}(\mu)\left(Y_{d, N}^{q}\right)^{T} \underline{y}_{N}+\frac{1}{2} \sum_{p=1}^{Q_{y d}} \sum_{q=1}^{Q_{y d}} \Theta_{y d}^{p}(\mu) \Theta_{y d}^{q}(\mu)\left(\tilde{Y}_{d}\right)_{p q}+\frac{\lambda}{2}\left(u_{N}-u_{d}\right)^{T}\left(u_{N}-u_{d}\right)
$$

in $\mathcal{O}\left(N^{2}+Q_{y d} N+Q_{y d}^{2}+m\right)$ operations. Hence, the computational cost for the online stage is independent of $\mathcal{N}$, the dimension of the underlying "truth" finite element approximation space. Since $N \ll \mathcal{N}$, we expect significant computational savings in the online stage relative to the solution of (3.6). However, we need to rigorously and efficiently assess the error introduced.

\section{A POSTERIORI ERROR ESTIMATION}

We will now develop a posteriori error bounds for several quantities of interest: the error in the optimal control, the error in the associated cost functional, and the error in a given linear output functional of the optimal solution.

We start with the control and cost functional bounds in Sections 4.1 and 4.2, respectively. In Section 4.3 we then propose a dual approach which considerably improves the convergence rate of our control error bound. The error bounds introduced are rigorous upper bounds for the true errors and are efficient to compute; we summarize the computational procedure in Section 4.4. Finally, we present the Greedy procedure to generate $Y_{N}$ in Section 4.5.

\subsection{Error bound for the optimal control}

We first consider the error in the optimal control. Our derivation is an extension of the results for scalar controls in [8] and is based on the following result from [31] (see Thm. 4.11 in [31] for the proof).

Theorem 4.1. Let $u^{*}$ and $u_{N}^{*}$ be the optimal solutions to the truth and reduced basis optimal control problems $(\mathrm{P})$ and $\left(\mathrm{P}_{\mathrm{N}}\right)$, respectively. The error in the optimal control then satisfies

$$
\left\|u^{*}-u_{N}^{*}\right\|_{\mathcal{U}} \leq \frac{1}{\lambda}\left\|\lambda\left(u_{N}^{*}-u_{d}\right)-\mathcal{B}^{\star} p\left(y\left(u_{N}^{*}\right)\right)\right\|_{\mathcal{U}}, \quad \forall \mu \in \mathcal{D} .
$$

We note that $y\left(u_{N}^{*}\right)$ is the solution of the (truth) state equation (3.6a) with control $u_{N}^{*}$ instead of $u^{*}$, and $p\left(y\left(u_{N}^{*}\right)\right)$ is the solution of the (truth) adjoint equation (3.6b) with $y\left(u_{N}^{*}\right)$ instead of $y^{*}\left(u^{*}\right)$ on the right-hand side. Evaluation of the bound (4.1) thus requires a solution of both truth approximations and is computationally expensive. In contrast, our new bound is online-efficient, i.e., its evaluation is independent of $\mathcal{N}$. The underlying idea is to replace the truth approximation $p\left(y\left(u_{N}^{*}\right)\right)$ in (4.1) with the reduced basis approximation $p_{N}^{*}\left(y_{N}^{*}\left(u_{N}^{*}\right)\right)$ and to bound the error term $p\left(y\left(u_{N}^{*}\right)\right)-p_{N}^{*}\left(y_{N}^{*}\left(u_{N}^{*}\right)\right)$.

Before we continue, let us make some notational remarks. Following the notation and terminology in [4], we refer to $\tilde{e}^{y}=y\left(u_{N}^{*}\right)-y_{N}^{*}\left(u_{N}^{*}\right)$ as the state predictability error and to $\tilde{e}^{p}=p\left(y\left(u_{N}^{*}\right)\right)-p_{N}^{*}\left(y_{N}^{*}\left(u_{N}^{*}\right)\right)$ as the adjoint predictability error. They reflect the ability of the corresponding reduced basis solutions to approximate the truth state and adjoint solutions for a prescribed control. In contrast, we define the state, adjoint, and control 
optimality errors as $e^{y, *}=y^{*}\left(u^{*}\right)-y_{N}^{*}\left(u_{N}^{*}\right), e^{p, *}=p^{*}\left(y^{*}\left(u^{*}\right)\right)-p_{N}^{*}\left(y_{N}^{*}\left(u_{N}^{*}\right)\right)$, and $e^{u, *}=u^{*}-u_{N}^{*}$, respectively. We start with the following definition.

Definition 4.2. The residuals of the state equation, the adjoint equation, and the optimality condition are defined by

$$
\begin{array}{lll}
r_{y}(\phi ; \mu)=\left\langle\mathcal{B} u_{N}^{*}, \phi\right\rangle_{Y^{\prime}, Y}-a\left(y_{N}^{*}, \phi ; \mu\right), & \forall \phi \in Y, & \forall \mu \in \mathcal{D}, \\
r_{p}(\varphi ; \mu)=\left(y_{d}(\mu)-y_{N}^{*}, \varphi\right)_{\mathrm{L}^{2}(D)}-a\left(\varphi, p_{N}^{*} ; \mu\right), & \forall \varphi \in Y, & \forall \mu \in \mathcal{D}, \\
r_{u}(\psi ; \mu)=\left(\lambda\left(u_{N}^{*}-u_{d}\right)-\mathcal{B}^{\star} p_{N}^{*}, \psi\right) \mathcal{U}, & \forall \psi \in \mathcal{U}, \quad \forall \mu \in \mathcal{D} .
\end{array}
$$

Note that since we do not reduce the already low-dimensional control space $\mathcal{U}$, the residual of the optimality condition vanishes, i.e., we in fact have $r_{u}(\psi ; \mu)=0, \forall \psi \in \mathcal{U}$.

Before stating the main result we require the following two intermediate results.

Lemma 4.3. The state predictability error, $\tilde{e}^{y}=y\left(u_{N}^{*}\right)-y_{N}^{*}\left(u_{N}^{*}\right)$, is bounded by

$$
\left\|\tilde{e}^{y}\right\|_{Y} \leq \tilde{\Delta}_{N}^{y}(\mu) \equiv \frac{\left\|r_{y}(\cdot ; \mu)\right\|_{Y^{\prime}}}{\alpha_{\mathrm{LB}}(\mu)}, \quad \forall \mu \in \mathcal{D}
$$

where $r_{y}(\phi ; \mu)$ is the state residual defined in $(4.2), y_{N}^{*}\left(u_{N}^{*}\right)$ is the solution of $(3.8 \mathrm{a})$, and $y\left(u_{N}^{*}\right)$ is the solution of the truth state equation (3.6a) with control $u_{N}^{*}$.

This is the standard a posteriori error bound for coercive elliptic PDEs, $c f$. Theorem 2.1 for the proof.

Lemma 4.4. The adjoint predictability error, $\tilde{e}^{p}=p\left(y\left(u_{N}^{*}\right)\right)-p_{N}^{*}\left(y_{N}^{*}\left(u_{N}^{*}\right)\right)$, is bounded by

$$
\left\|\tilde{e}^{p}\right\|_{Y} \leq \tilde{\Delta}_{N}^{p}(\mu) \equiv \frac{1}{\alpha_{\mathrm{LB}}(\mu)}\left(\left\|r_{p}(\cdot ; \mu)\right\|_{Y^{\prime}}+C_{D}^{2} \tilde{\Delta}_{N}^{y}(\mu)\right), \quad \forall \mu \in \mathcal{D}
$$

where $r_{p}(\varphi ; \mu)$ is the adjoint residual defined in (4.3), $C_{D} \equiv \sup _{v \in Y \backslash\{0\}} \frac{\|v\|_{L^{2}(D)}}{\|v\|_{Y}}, p_{N}^{*}\left(y_{N}^{*}\left(u_{N}^{*}\right)\right)$ is the solution of $(3.8 \mathrm{~b})$, and $p\left(y\left(u_{N}^{*}\right)\right)$ is the solution of the truth adjoint equation (3.6b) with $y\left(u_{N}^{*}\right)$ on the right-hand side.

Proof. We note from the definition of the adjoint residual, $r_{p}(\varphi ; \mu)$, and $(3.6 \mathrm{~b})$ that the error, $\tilde{e}^{p}$, satisfies

$$
a\left(\varphi, \tilde{e}^{p} ; \mu\right)=r_{p}(\varphi ; \mu)+\left(y_{N}^{*}\left(u_{N}^{*}\right)-y\left(u_{N}^{*}\right), \varphi\right)_{\mathrm{L}^{2}(D)}, \quad \forall \varphi \in Y .
$$

We now choose $\varphi=\tilde{e}^{p}$, invoke (2.7), (2.10), the definition of the dual norm of the residual, and the Cauchy-Schwarz inequality to obtain

$$
\alpha_{\mathrm{LB}}(\mu)\left\|\tilde{e}^{p}\right\|_{Y}^{2} \leq\left\|r_{p}(\cdot ; \mu)\right\|_{Y^{\prime}}\left\|\tilde{e}^{p}\right\|_{Y}+\left\|y\left(u_{N}^{*}\right)-y_{N}^{*}\left(u_{N}^{*}\right)\right\|_{\mathrm{L}^{2}(D)}\left\|\tilde{e}^{p}\right\|_{\mathrm{L}^{2}(D)}
$$

The desired result directly follows from the definition of $C_{D}$ and Lemma 4.3.

We note that this proof is in fact a simple extension of the proof of Theorem 2.1. The main difference is the additional error term due to the change in the right-hand sides of equations $(3.6 \mathrm{~b})$ and $(3.8 \mathrm{~b})$. This error in the right-hand side propagates and results in the additional term in the error bound (4.6).

We are now ready to state the optimal control error bound in

Proposition 4.5. Let $u^{*}$ and $u_{N}^{*}$ be the optimal solutions to the truth and reduced basis optimal control problems, respectively. Given $\tilde{\Delta}_{N}^{p}(\mu)$ defined in (4.6), the error in the optimal control satisfies

$$
\left\|u^{*}-u_{N}^{*}\right\|_{\mathcal{U}} \leq \Delta_{N}^{u, *}(\mu) \equiv \frac{1}{\lambda}\left(\sum_{i=1}^{m}\left\|b_{i}\right\|_{Y^{\prime}}^{2}\right)^{1 / 2} \tilde{\Delta}_{N}^{p}(\mu), \quad \forall \mu \in \mathcal{D} .
$$


Proof. We append $\pm \mathcal{B}^{\star} p_{N}^{*}\left(y_{N}^{*}\left(u_{N}^{*}\right)\right)$ to the bound in (4.1) and invoke (3.8c) to obtain

$$
\left\|u^{*}-u_{N}^{*}\right\|_{\mathcal{U}} \leq \frac{1}{\lambda}\left\|\mathcal{B}^{\star}\left(p\left(y\left(u_{N}^{*}\right)\right)-p_{N}^{*}\left(y_{N}^{*}\left(u_{N}^{*}\right)\right)\right)\right\|_{\mathcal{U}}, \quad \forall \mu \in \mathcal{D} .
$$

Furthermore, from the definition of the dual operator $\mathcal{B}^{\star}$ and the dual norm of $b_{1}, \ldots, b_{m}$ we have

$$
\frac{1}{\lambda}\left\|\mathcal{B}^{\star}\left(p\left(y\left(u_{N}^{*}\right)\right)-p_{N}^{*}\left(y_{N}^{*}\left(u_{N}^{*}\right)\right)\right)\right\|_{\mathcal{U}}=\frac{1}{\lambda}\left(\sum_{i=1}^{m} b_{i}\left(\tilde{e}^{p}\right)^{2}\right)^{1 / 2} \leq \frac{1}{\lambda}\left(\sum_{i=1}^{m}\left\|b_{i}\right\|_{Y^{\prime}}^{2}\right)^{1 / 2}\left\|\tilde{e}^{p}\right\|_{Y} .
$$

The desired result directly follows from Lemma 4.4.

\subsection{Error bound for the cost functional}

Given the error bound $\Delta_{N}^{u, *}(\mu)$ for the optimal control we may readily derive a bound for the error in the cost functional. We require the following two preparatory lemmata stating the a posteriori error bounds for the state and adjoint optimality errors. We note that the proofs of these lemmata are similar to the proof of Lemma 4.4, i.e., the error in the optimal control - or, more precisely, the error bound of the optimal control propagates and appears as an additional term in the state and adjoint optimality error bound.

Lemma 4.6. The state optimality error, $e^{y, *}=y^{*}\left(u^{*}\right)-y_{N}^{*}\left(u_{N}^{*}\right)$, is bounded by

$$
\left\|e^{y, *}\right\|_{Y} \leq \Delta_{N}^{y, *}(\mu) \equiv \frac{1}{\alpha_{\mathrm{LB}}(\mu)}\left(\left\|r_{y}(\cdot ; \mu)\right\|_{Y^{\prime}}+\left(\sum_{i=1}^{m}\left\|b_{i}\right\|_{Y^{\prime}}^{2}\right)^{\frac{1}{2}} \Delta_{N}^{u, *}(\mu)\right), \quad \forall \mu \in \mathcal{D} .
$$

Proof. We note from (4.2) and (3.6a) that the error, $e^{y, *}$, satisfies

$$
a\left(e^{y, *}, \phi ; \mu\right)=r_{y}(\phi ; \mu)+\sum_{i=1}^{m} b_{i}(\phi)\left(u_{i}^{*}-u_{N i}^{*}\right), \quad \forall \phi \in Y .
$$

We now choose $\phi=e^{y, *}$, invoke (2.7), (2.10), the definition of the dual norm of the residual and the linear functionals $b_{1}, \ldots, b_{m}$ to obtain

$$
\alpha_{\mathrm{LB}}(\mu)\left\|e^{y, *}\right\|_{Y}^{2} \leq\left\|r_{y}(\cdot ; \mu)\right\|_{Y^{\prime}}\left\|e^{y, *}\right\|_{Y}+\left\|e^{y, *}\right\|_{Y} \sum_{i=1}^{m}\left\|b_{i}\right\|_{Y^{\prime}}\left|u_{i}^{*}-u_{N i}^{*}\right| .
$$

We use the Cauchy-Schwarz inequality and invoke Proposition 4.5 to obtain the desired result.

Lemma 4.7. The adjoint optimality error, $e^{p, *}=p^{*}\left(y^{*}\left(u^{*}\right)\right)-p_{N}^{*}\left(y_{N}^{*}\left(u_{N}^{*}\right)\right)$, is bounded by

$$
\left\|e^{p, *}\right\|_{Y} \leq \Delta_{N}^{p, *}(\mu) \equiv \frac{1}{\alpha_{\mathrm{LB}}(\mu)}\left(\left\|r_{p}(\cdot ; \mu)\right\|_{Y^{\prime}}+C_{D}^{2} \Delta_{N}^{y, *}(\mu)\right), \quad \forall \mu \in \mathcal{D} .
$$

Proof. We note from (4.3) and (3.6b) that the error, $e^{p, *}$, satisfies

$$
a\left(\varphi, e^{p, *} ; \mu\right)=r_{p}(\varphi ; \mu)+\left(y_{N}^{*}\left(u_{N}^{*}\right)-y^{*}\left(u^{*}\right), \varphi\right)_{\mathrm{L}^{2}(D)}, \quad \forall \varphi \in Y .
$$

We now choose $\varphi=e^{p, *}$, invoke (2.7), (2.10), the definition of the dual norm of the residual, and the Cauchy-Schwarz inequality to obtain

$$
\alpha_{\mathrm{LB}}(\mu)\left\|e^{p, *}\right\|_{Y}^{2} \leq\left\|r_{p}(\cdot ; \mu)\right\|_{Y^{\prime}}\left\|e^{p, *}\right\|_{Y}+\left\|y^{*}\left(u^{*}\right)-y_{N}^{*}\left(u_{N}^{*}\right)\right\|_{\mathrm{L}^{2}(D)}\left\|e^{p, *}\right\|_{\mathrm{L}^{2}(D)} .
$$

The desired result directly follows from the definition of $C_{D}$ and Lemma 4.6. 
We can now state

Proposition 4.8. Let $J^{*}=J\left(y^{*}, u^{*} ; \mu\right)$ and $J_{N}^{*}=J\left(y_{N}^{*}, u_{N}^{*} ; \mu\right)$ be the optimal values of the cost functionals of the truth and reduced basis optimal control problems, respectively. The error then satisfies

$$
\left|J^{*}-J_{N}^{*}\right| \leq \Delta_{N}^{J, *}(\mu) \equiv \frac{1}{2}\left(\left\|r_{y}(\cdot ; \mu)\right\|_{Y^{\prime}} \Delta_{N}^{p, *}(\mu)+\left\|r_{p}(\cdot ; \mu)\right\|_{Y^{\prime}} \Delta_{N}^{y, *}(\mu)\right), \quad \forall \mu \in \mathcal{D} .
$$

Proof. We use the standard result from [2] to estimate the error in the cost functional by

$$
\left|J^{*}-J_{N}^{*}\right| \leq \frac{1}{2}\left(\left\|r_{y}(\cdot ; \mu)\right\|_{Y^{\prime}}\left\|e^{p, *}\right\|_{Y}+\left\|r_{p}(\cdot ; \mu)\right\|_{Y^{\prime}}\left\|e^{y, *}\right\|_{Y}\right), \quad \forall \mu \in \mathcal{D} .
$$

The result follows from Lemma 4.6 and 4.7.

We briefly remark at this point that our a posteriori bounds defined in (4.9) and (4.18) are rigorous upper bounds for the true errors and that the standard reduced basis offline-online computational procedure directly applies - online evaluation of $\Delta_{N}^{u, *}(\mu)$ and $\Delta_{N}^{J, *}(\mu)$ is independent of $\mathcal{N}$; see Section 4.4.

\subsection{Duality based error bound}

In this section we propose superconvergent a posteriori error bounds for linear output functionals of the state, adjoint, and control by employing dual techniques. Since each control component $u_{1}, \ldots, u_{m}$ is a linear output of the optimal solution $x^{*}=\left(y^{*}, p^{*}, u^{*}\right)$, this approach allows us to considerably improve the control error bound (4.9) - and subsequently also the cost functional error bound (4.18). Dual techniques are widely used in PDE approximations to obtain superlinear convergence of linear output functionals [24]; also see for example $[3,23]$ in the finite element context. These ideas have also been used successfully to improve the convergence of reduced basis output approximations and the associated a posteriori error bounds in $[10,25,32]$.

We note from (4.18) that the error bound for the cost functional $\Delta_{N}^{J, *}(\mu)$ is in fact superconvergent, i.e., $\Delta_{N}^{J, *}(\mu)$ is proportional to the product of error terms involving the state and adjoint solutions of the optimality system. The control error bound $\Delta_{N}^{u, *}(\mu)$ defined in (4.9), on the other hand, is only proportional to the error in the adjoint solution. If the actual error in the control converges superlinearly, the effectivities, i.e., the ratio of the error bound and the actual error, will thus grow with the dimension $N$ of the reduced basis approximation. We will observe and comment on this behavior in Section 5; also see the results in [8].

Our goal is to avoid this growth of the effectivity with $N$ by employing dual techniques. We first derive the theory for a general linear output functional of the optimal solution $x^{*}=\left(y^{*}, p^{*}, u^{*}\right)$ and then state the results for the improved control and cost functional error bounds.

\subsubsection{Linear output functional}

We start by writing the truth optimality system (3.6a)-(3.6c) in a more condensed form. To this end, we introduce the product space $\mathcal{X}=Y \times Y \times \mathcal{U}$ and define, for any $\mu \in \mathcal{D}$, the bilinear form $h(\cdot, \cdot ; \mu): \mathcal{X} \times \mathcal{X} \rightarrow \mathbb{R}$ as

$$
\begin{aligned}
h(x, z ; \mu) & =h((y, p, u),(\phi, \varphi, \psi) ; \mu) \\
& =a(y, \phi ; \mu)-\langle\mathcal{B} u, \phi\rangle_{Y^{\prime}, Y}+a(\varphi, p ; \mu)+(y, \varphi)_{\mathrm{L}^{2}(D)}+\left(\lambda u-\mathcal{B}^{\star} p, \psi\right)_{\mathcal{U}} .
\end{aligned}
$$

The optimality system (3.6a)-(3.6c) can then be written compactly as: Given $\mu \in \mathcal{D}$, the optimal solution $x^{*}=\left(y^{*}, p^{*}, u^{*}\right) \in \mathcal{X}$ satisfies

$$
h\left(x^{*}, z ; \mu\right)=f(z), \quad \forall z \in \mathcal{X},
$$

where $z=(\phi, \varphi, \psi)$ and the bounded linear functional $f \in \mathcal{X}^{\prime}$ is defined as

$$
f(z)=\left(y_{d}(\mu), \varphi\right)_{\mathrm{L}^{2}(D)}+\lambda\left(u_{d}, \psi\right)_{\mathcal{U}} .
$$

We are interested in the evaluation of a linear output $\ell\left(x^{*}\right)$ of the optimal solution $x^{*}=\left(y^{*}, p^{*}, u^{*}\right)$. Here, $\ell \in \mathcal{X}^{\prime}$ is a bounded linear output functional which is composed of its components $\ell_{y}, \ell_{p} \in Y^{\prime}$ and $\ell_{u} \in \mathcal{U}^{\prime}$ and 
is given by $\ell(z)=\ell_{y}(\phi)+\ell_{p}(\varphi)+\ell_{u}(\psi)$. Note that we obtain the control component $u_{i}$ as output by simply choosing $\ell_{y}(\phi)=\ell_{p}(\varphi)=0$ and $\ell_{u}(\psi)=\psi_{i}$; similarly, we can set $\ell_{p}(\varphi)=\ell_{u}(\psi)=0$ and $\ell_{y}(\phi) \neq 0$ if we are interested in a linear output of the state $y$.

We can also directly state the reduced basis optimality system (3.8) in this form: Given $\mu \in \mathcal{D}$, find $x_{N}^{*}=$ $\left(y_{N}^{*}, p_{N}^{*}, u_{N}^{*}\right) \in \mathcal{X}_{N}=Y_{N} \times Y_{N} \times \mathcal{U}$ such that

$$
h\left(x_{N}^{*}, z ; \mu\right)=f(z), \quad \forall z \in \mathcal{X}_{N}
$$

we then obtain the reduced basis output approximation from $\ell\left(x_{N}^{*}\right)$.

We next introduce the associated truth dual problem [24]: Given $\mu \in \mathcal{D}$, the dual variable $\hat{x}^{*}=\left(\hat{y}^{*}, \hat{p}^{*}, \hat{u}^{*}\right) \in \mathcal{X}$ satisfies

$$
h\left(z, \hat{x}^{*} ; \mu\right)=\ell(z), \quad \forall z \in \mathcal{X} .
$$

The left-hand side of the dual problem (4.24) has the form

$$
h\left(z, \hat{x}^{*} ; \mu\right)=a\left(\phi, \hat{y}^{*} ; \mu\right)-\left\langle\mathcal{B} \psi, \hat{y}^{*}\right\rangle_{Y^{\prime}, Y}+a\left(\hat{p}^{*}, \varphi ; \mu\right)+\left(\phi, \hat{p}^{*}\right)_{\mathrm{L}^{2}(D)}+\left(\lambda \psi-\mathcal{B}^{\star} \varphi, \hat{u}^{*}\right) \mathcal{U},
$$

and - by sorting with respect to the test functions - the dual problem (4.24) can thus be written in extended form as

$$
\begin{aligned}
a\left(\phi, \hat{y}^{*} ; \mu\right)+\left(\phi, \hat{p}^{*}\right)_{\mathrm{L}^{2}(D)} & =\ell_{y}(\phi), & & \forall \phi \in Y, \\
a\left(\hat{p}^{*}, \varphi ; \mu\right)-\left\langle\mathcal{B} \hat{u}^{*}, \varphi\right\rangle_{Y^{\prime}, Y} & =\ell_{p}(\varphi), & & \forall \varphi \in Y, \\
\left(\lambda \hat{u}^{*}-\mathcal{B}^{\star} \hat{y}^{*}, \psi\right)_{\mathcal{U}} & =\ell_{u}(\psi), & & \forall \psi \in \mathcal{U} .
\end{aligned}
$$

We note that the variables $\hat{y}$ and $\hat{p}$ in the dual optimality system have switched the roles compared to $y$ and $p$ in the primal optimality system. We thus will refer to (4.26b) as the dual state equation and to (4.26a) as the dual adjoint equation.

At this point we should in fact remark on a conflict in notation. Although the expressions "dual" and "adjoint" are often used interchangeably, we need to distinguish between the two, i.e., we refer to (3.6) as the primal optimality system containing the primal state equation, primal adjoint equation, and primal optimality condition, and to (4.26) as the dual optimality system containing the dual state equation, dual adjoint equation, and dual optimality condition.

It remains to introduce the reduced basis approximation of the dual problem (4.24). In analogy to the primal problem we introduce the "integrated" dual reduced basis spaces

$$
\hat{Y}_{M}=\operatorname{span}\left\{\zeta_{m}^{\mathrm{du}}, 1 \leq m \leq M\right\}=\operatorname{span}\left\{\hat{y}^{*}\left(\mu_{\mathrm{du}}^{m}\right), \hat{p}^{*}\left(\mu_{\mathrm{du}}^{m}\right), 1 \leq m \leq M / 2\right\}, \quad 1 \leq M / 2 \leq M_{\max } / 2,
$$

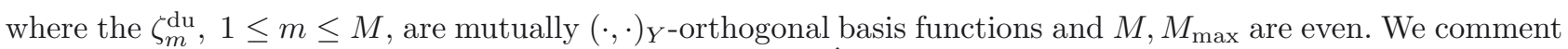
on the Greedy sampling procedure to construct the spaces $\hat{Y}_{M}$ in Section 4.5. The dual reduced basis optimality system is then: Given $\mu \in \mathcal{D}$, find $\hat{x}_{M}^{*}=\left(\hat{y}_{M}^{*}, \hat{p}_{M}^{*}, \hat{u}_{M}^{*}\right) \in \hat{\mathcal{X}}_{M}=\hat{Y}_{M} \times \hat{Y}_{M} \times \mathcal{U}$ such that

$$
\begin{aligned}
a\left(\phi, \hat{y}_{M}^{*} ; \mu\right)+\left(\phi, \hat{p}_{M}^{*}\right)_{L^{2}(D)} & =\ell_{y}(\phi), & & \forall \phi \in \hat{Y}_{M}, \\
a\left(\hat{p}_{M}^{*}, \varphi ; \mu\right)-\left\langle\mathcal{B} \hat{u}_{M}^{*}, \varphi\right\rangle_{Y^{\prime}, Y} & =\ell_{p}(\varphi), & & \forall \varphi \in \hat{Y}_{M}, \\
\left(\lambda \hat{u}_{M}^{*}-\mathcal{B}^{\star} \hat{y}_{M}^{*}, \psi\right)_{\mathcal{U}} & =\ell_{u}(\psi), & & \forall \psi \in \mathcal{U} .
\end{aligned}
$$

We can now prove the following bound for the error in the output functional.

Theorem 4.9. Let $x^{*} \in \mathcal{X}$ and $x_{N}^{*} \in \mathcal{X}_{N}$ be the optimal solutions to the primal truth and reduced basis optimality systems (3.6) and (3.8), respectively. Furthermore, let $\hat{x}^{*} \in \mathcal{X}$ and $\hat{x}_{M}^{*} \in \hat{\mathcal{X}}_{M}$ be the optimal solutions to the dual truth and reduced basis optimality systems (4.26) and (4.28), respectively. The error in the output functional $\ell$ then satisfies

$$
\begin{gathered}
\left|\ell\left(x^{*}\right)-\ell\left(x_{N}^{*}\right)\right| \leq\left\|r_{y}(\cdot ; \mu)\right\|_{Y^{\prime}}\left\|\hat{y}^{*}-\hat{y}_{M}^{*}\right\|_{Y} \quad+\left\|r_{p}(\cdot ; \mu)\right\|_{Y^{\prime}}\left\|\hat{p}^{*}-\hat{p}_{M}^{*}\right\|_{Y} \\
+\left|r_{y}\left(\hat{y}_{M}^{*} ; \mu\right)+r_{p}\left(\hat{p}_{M}^{*} ; \mu\right)\right|, \quad \forall \mu \in \mathcal{D} .
\end{gathered}
$$


Proof. From the linearity of the output functional $\ell$ and the definition of the dual problem (4.24) we obtain

$$
\ell\left(x^{*}\right)-\ell\left(x_{N}^{*}\right)=\ell\left(x^{*}-x_{N}^{*}\right)=h\left(x^{*}-x_{N}^{*}, \hat{x}^{*} ; \mu\right), \quad \forall \mu \in \mathcal{D} .
$$

Furthermore, for every $z=(\phi, \varphi, \psi)^{T} \in \mathcal{X}$ the error residual relationship

$$
\begin{aligned}
h\left(x^{*}-x_{N}^{*}, z ; \mu\right) & =f(z)-h\left(x_{N}^{*}, z ; \mu\right) \\
& =r_{y}(\phi)+r_{p}(\varphi)+r_{u}(\psi)=r_{y}(\phi)+r_{p}(\varphi)
\end{aligned}
$$

holds by Definition 4.2. We now choose $z=\hat{x}^{*}$ and obtain

$$
\begin{aligned}
h\left(x^{*}-x_{N}^{*}, \hat{x}^{*} ; \mu\right) & =h\left(x^{*}-x_{N}^{*}, \hat{x}^{*}-\hat{x}_{M}^{*} ; \mu\right)+h\left(x^{*}-x_{N}^{*}, \hat{x}_{M}^{*} ; \mu\right) \\
& =r_{y}\left(\hat{y}^{*}-\hat{y}_{M}^{*} ; \mu\right)+r_{p}\left(\hat{p}^{*}-\hat{p}_{M}^{*} ; \mu\right)+r_{y}\left(\hat{y}_{M}^{*} ; \mu\right)+r_{p}\left(\hat{p}_{M}^{*} ; \mu\right) .
\end{aligned}
$$

The result now follows directly from the definition of the dual norms of the residuals.

Note that $r_{y}$ and $r_{p}$ are the residuals associated to the primal optimality system and that their dual norms $\left\|r_{y}(\cdot ; \mu)\right\|_{Y^{\prime}}$ and $\left\|r_{p}(\cdot ; \mu)\right\|_{Y^{\prime}}$ as well as the residual correction terms $r_{y}\left(\hat{y}_{M}^{*} ; \mu\right)$ and $r_{p}\left(\hat{p}_{M}^{*} ; \mu\right)$ can be evaluated efficiently using an offline-online decomposition; see Section 4.4. However, we still need to develop efficiently evaluable a posteriori error bounds for the dual optimality errors $\hat{y}^{*}-\hat{y}_{M}^{*}$ and $\hat{p}^{*}-\hat{p}_{M}^{*}$. Fortunately, the derivation of these bounds follows directly from the analysis in Sections 4.1 and 4.2: we first derive bounds for the dual predictability errors and the dual optimal "control" $\hat{u}^{*}-\hat{u}_{M}^{*}$; we then employ the dual control error bound to obtain the dual optimality error bounds. We summarize the necessary results in Appendix 5. Given the dual optimality error bounds, we can state the main result of this section.

Proposition 4.10. Let $x^{*}$ and $x_{N}^{*}$ be the optimal solutions to the primal truth and reduced basis optimality systems, respectively. Furthermore, let $\hat{x}^{*}$ and $\hat{x}_{M}^{*}$ be the optimal solutions to the dual truth and reduced basis optimality systems, respectively. The error in the output functional $\ell$ then satisfies

$$
\left|\ell\left(x^{*}\right)-\ell\left(x_{N}^{*}\right)\right| \leq \Delta_{N, M}^{\ell, *}(\mu), \quad \forall \mu \in \mathcal{D},
$$

where

$$
\Delta_{N, M}^{\ell, *}(\mu) \equiv\left\|r_{y}(\cdot ; \mu)\right\|_{Y^{\prime}} \Delta_{M}^{\hat{y}, *}(\mu)+\left\|r_{p}(\cdot ; \mu)\right\|_{Y^{\prime}} \Delta_{M}^{\hat{p}, *}(\mu)+\left|r_{y}\left(\hat{y}_{M}^{*} ; \mu\right)+r_{p}\left(\hat{p}_{M}^{*} ; \mu\right)\right|, \quad \forall \mu \in \mathcal{D} .
$$

Proof. The result follows directly from Theorem 4.9 and the definitions of the dual state and adjoint optimality error bounds $\Delta_{M}^{\hat{p}, *}(\mu)$ and $\Delta_{M}^{\hat{y}, *}(\mu)$ in Lemmas A.5 and A.6, respectively.

The primal-dual error bound $\Delta_{N, M}^{\ell, *}(\mu)$ obviously depends on the dimensions of the primal and dual reduced basis approximations through the primal residuals and the dual optimal solutions and associated bounds. We present the computational procedure to evaluate $\Delta_{N, M}^{\ell, *}(\mu)$ in Section 4.4.

\subsubsection{Optimal control}

We may employ the dual approach to obtain superlinear convergent a posteriori error bounds for the optimal control. To this end, we consider the linear output functional $\ell^{u_{i}}(z)=\psi_{i}$ for $z=(\phi, \varphi, \psi) \in \mathcal{X}$ associated to the $i$-th control component $u_{i}$. The error in the $i$-th control component then coincides with the error for the functional $\ell^{u_{i}}$ and is thus bounded by

$$
\left|u_{i}^{*}-u_{N i}^{*}\right|=\left|\ell^{u_{i}}\left(x^{*}\right)-\ell^{u_{i}}\left(x_{N}^{*}\right)\right| \leq \Delta_{N, M}^{\ell_{i}, *}(\mu) .
$$

Consequently, for the error in the optimal control we directly obtain 
Corollary 4.11. Let $x^{*}$ and $x_{N}^{*}$ be the optimal solutions to the primal truth and reduced basis optimal control problems, respectively. The error in the optimal control then satisfies

$$
\left\|u^{*}-u_{N}^{*}\right\|_{\mathcal{U}} \leq \Delta_{N, M}^{u, *}(\mu) \equiv\left(\sum_{i=1}^{m}\left(\Delta_{N, M}^{\ell_{i}^{u_{i}, *}}(\mu)\right)^{2}\right)^{\frac{1}{2}} .
$$

We note that the evaluation of the primal-dual error bound (4.38) requires $m$ dual problems in addition to the primal optimal control problem and is thus feasible only for problems involving a modest number of controls.

\subsubsection{Cost functional}

We mentioned in the beginning of Section 4.3 that the (primal-only) error bound for the cost functional $\Delta_{N}^{J, *}(\mu)$ defined in (4.18) is already superconvergent. However, the cost error bound can still benefit indirectly from the dual approach. As pointed out in Section 4.2, the control error bound propagates and enters as an additional error term in the state and adjoint optimality error bounds. A sharper (primal-dual) control error bound will hence lead to sharper optimality error bounds and finally to a sharper error bound for the cost functional.

The approach is as follows: we replace the control error bound $\Delta_{N}^{u, *}(\mu)$ in (4.12) with its primal-dual counterpart $\Delta_{N, M}^{u, *}(\mu)$ to obtain the primal-dual state optimality error bound

$$
\Delta_{N, M}^{y, *}(\mu) \equiv \frac{1}{\alpha_{\mathrm{LB}}(\mu)}\left(\left\|r_{y}(\cdot ; \mu)\right\|_{Y^{\prime}}+\left(\sum_{i=1}^{m}\left\|b_{i}\right\|_{Y^{\prime}}^{2}\right)^{\frac{1}{2}} \Delta_{N, M}^{u, *}(\mu)\right)
$$

and subsequently the primal-dual adjoint optimality error bound

$$
\Delta_{N, M}^{p, *}(\mu) \equiv \frac{1}{\alpha_{\mathrm{LB}}(\mu)}\left(\left\|r_{p}(\cdot ; \mu)\right\|_{Y^{\prime}}+C_{D}^{2} \Delta_{N, M}^{y, *}(\mu)\right) .
$$

These bounds in turn allow to develop a new primal-dual cost functional error bound given by

$$
\Delta_{N, M}^{J, *}(\mu) \equiv \frac{1}{2}\left(\left\|r_{y}(\cdot ; \mu)\right\|_{Y^{\prime}} \Delta_{N, M}^{y, *}(\mu)+\left\|r_{p}(\cdot ; \mu)\right\|_{Y^{\prime}} \Delta_{N, M}^{p, *}(\mu)\right)
$$

Here, the primal-only state and adjoint optimality error bounds $\Delta_{N}^{y, *}(\mu)$ and $\Delta_{N}^{p, *}(\mu)$ in (4.18) have been replaced by $\Delta_{N, M}^{y, *}(\mu)$ and $\Delta_{N, M}^{p, *}(\mu)$, respectively.

\subsection{Computational procedure}

\subsubsection{Control and cost functional error bound}

For the evaluation of the control and cost functional error bounds described in Section 4.1 and 4.2 the following quantities need to be computed: the dual norms of the state and adjoint equation residuals $\left\|r_{y}(\cdot ; \mu)\right\|_{Y^{\prime}}$ and $\left\|r_{p}(\cdot ; \mu)\right\|_{Y^{\prime}}$, respectively; the constant $C_{D}$; the dual norms of the linear functionals $\left\|b_{i}\right\|_{Y^{\prime}}, 1 \leq i \leq m$; and the coercivity lower bound $\alpha_{\mathrm{LB}}(\mu)$. Since all of these quantities can be evaluated using the standard offline-online decomposition [29], we only summarize the computational cost in the online stage. Given a new parameter $\mu \in \mathcal{D}$ and associated optimal solution $x_{N}^{*}$, evaluation of $\Delta_{N}^{u, *}(\mu)$ and $\Delta_{N}^{J, *}(\mu)$ requires (to leading order) $\mathcal{O}\left(\left(Q_{a} N+m\right)^{2}+\left(Q_{a} N+N+Q_{y d}\right)^{2}\right)$ operations, and is thus independent of $\mathcal{N}$.

\subsubsection{Dual problem}

The computational procedure for the reduced basis approximation of the dual optimality system is analogous to the one for the primal problem. We express the reduced basis dual state and adjoint solution as $\hat{y}_{M}(\mu)=\sum_{i=1}^{M} \hat{y}_{M i}(\mu) \zeta_{i}^{\mathrm{du}}$ and $\hat{p}_{M}(\mu)=\sum_{i=1}^{M} \hat{p}_{M i}(\mu) \zeta_{i}^{\mathrm{du}}$ and denote the coefficient vectors by $\underline{\hat{y}}_{M}(\mu)=\left[\hat{y}_{M 1}(\mu), \ldots, \hat{y}_{M M}(\mu)\right]^{T} \in \mathbb{R}^{M}$ and $\underline{\hat{p}}_{M}(\mu)=\left[\hat{p}_{M 1}(\mu), \ldots, \hat{p}_{M M}(\mu)\right]^{T} \in \mathbb{R}^{M}$, respectively. If we choose 
as test functions $\phi=\zeta_{i}^{\mathrm{du}}, 1 \leq i \leq M, \varphi=\zeta_{i}^{\mathrm{du}}, 1 \leq i \leq M$ and $\psi=e_{i}, 1 \leq i \leq m$ in (4.28), where $e_{i}$ denotes the $i$-th unit vector in $\mathbb{R}^{m}$, the dual reduced basis optimality system can be expressed in terms of the $(2 M+m) \times(2 M+m)$ linear system

$$
\left[\begin{array}{ccc}
A_{M}^{T}(\mu) & D_{M} & 0 \\
0 & A_{M}(\mu) & -B_{M} \\
-B_{M}^{T} & 0 & \lambda I_{m}
\end{array}\right]\left[\begin{array}{c}
\frac{\hat{y}}{\hat{p}_{M}} \\
\hat{\hat{u}}_{M}
\end{array}\right]=\left[\begin{array}{c}
L_{M}^{y} \\
L_{M}^{p} \\
L^{u}
\end{array}\right] .
$$

Here, $A_{M}(\mu) \in \mathbb{R}^{M \times M}, D_{M} \in \mathbb{R}^{M \times M}$, and $B_{M} \in \mathbb{R}^{M \times m}$ are matrices with the entries $\left(A_{M}(\mu)\right)_{i j}=$ $a\left(\zeta_{j}^{\mathrm{du}}, \zeta_{i}^{\mathrm{du}} ; \mu\right), 1 \leq i, j \leq M,\left(D_{M}\right)_{i j}=\left(\zeta_{j}^{\mathrm{du}}, \zeta_{i}^{\mathrm{du}}\right)_{\mathrm{L}^{2}(D)}, 1 \leq i, j \leq M$, and $\left(B_{M}\right)_{i j}=b_{j}\left(\zeta_{i}^{\mathrm{du}}\right), 1 \leq i \leq M, 1 \leq$ $j \leq m$, respectively. The vectors $L_{M}^{y} \in \mathbb{R}^{M}$ and $L_{M}^{p} \in \mathbb{R}^{M}$ are given by $\left(L_{M}^{y}\right)_{i}=\ell_{y}\left(\zeta_{i}^{\mathrm{du}}\right), 1 \leq i \leq M$, and $\left(L_{M}^{p}\right)_{i}=\ell^{p}\left(\zeta_{i}^{\mathrm{du}}\right), 1 \leq i \leq M$, respectively. We denote by $I_{m} \in \mathbb{R}^{m \times m}$ the identity matrix and $L^{u} \in \mathbb{R}^{m}$ is the Riesz representation of $\ell_{u}$. Invoking the affine parameter dependence (2.3) yields the ex-

pansion $A_{M}(\mu)=\sum_{q=1}^{Q_{a}} \Theta_{a}^{q}(\mu) A_{M}^{q}$, where the parameter-independent matrices $A_{M}^{q} \in \mathbb{R}^{M \times M}$ are given by $\left(A_{M}^{q}\right)_{i j}=a^{q}\left(\zeta_{j}^{\mathrm{du}}, \zeta_{i}^{\mathrm{du}}\right), 1 \leq i, j \leq M, 1 \leq q \leq Q_{a}$.

The offline-online decomposition is now clear. In the offline stage - performed only once - we first construct the reduced basis space $\hat{Y}_{M}$ and assemble the parameter-independent matrices $A_{M}^{q}, 1 \leq q \leq Q_{a}, D_{M}, B_{M}$, and the parameter-independent vectors $L_{M}^{y}, L_{M}^{p}$; the computational cost clearly dependents on $\mathcal{N}$. In the online stage - for each new parameter value $\mu$ - we first assemble the parameter-dependent matrix $A_{M}(\mu)$ in $\mathcal{O}\left(Q_{a} M^{2}\right)$ operations. We then solve the reduced basis optimality system $(4.42)$ at cost $\mathcal{O}\left((2 M+m)^{3}\right)$. Hence, the computational cost for the online stage is independent of $\mathcal{N}$.

\subsubsection{Duality based error bound}

For the evaluation of the primal-dual error bound $\Delta_{N, M}^{\ell, *}(\mu)$ in (4.36) we additionally need the following ingredients: the dual norms of the dual residuals $\left\|\hat{r}_{\hat{p}}(\cdot ; \mu)\right\|_{Y^{\prime}}$ and $\left\|\hat{r}_{\hat{y}}(\cdot ; \mu)\right\|_{Y^{\prime}}$ and the residual correction terms $r_{y}\left(\hat{y}_{M} ; \mu\right)$ and $r_{p}\left(\hat{p}_{M} ; \mu\right)$. Again, the offline-online decomposition for the dual norms of the residuals is standard; we thus focus on the residual correction terms.

In the offline stage, given $Y_{N}$ and $\hat{Y}_{M}$, we assemble the parameter-independent matrices $A_{N, M}^{q} \in \mathbb{R}^{N \times M}$, $A_{M, N}^{q} \in \mathbb{R}^{M \times N}$, and $D_{N, M} \in \mathbb{R}^{N \times M}$ with entries $\left(A_{N, M}^{q}\right)_{i j}=a^{q}\left(\zeta_{i}, \zeta_{j}^{\mathrm{du}}\right), 1 \leq i \leq N, 1 \leq j \leq M, 1 \leq q \leq Q_{a}$, $\left(A_{M, N}^{q}\right)_{i j}=a^{q}\left(\zeta_{i}^{\mathrm{du}}, \zeta_{j}\right), 1 \leq i \leq M, 1 \leq j \leq N, 1 \leq q \leq Q_{a}$, and $\left(D_{N, M}\right)_{i j}=\left(\zeta_{i}, \zeta_{j}^{\mathrm{du}}\right)_{\mathrm{L}^{2}(D)}, 1 \leq i \leq N, 1 \leq j \leq$ $M$, respectively; and the parameter-independent vector $Y_{d, M}^{q} \in \mathbb{R}^{M}$ with entries $\left(Y_{d, M}^{q}\right)_{i}=\left(y_{d}^{q}, \zeta_{i}^{\mathrm{du}}\right)_{\mathrm{L}^{2}(D)}, 1 \leq$ $i \leq M, 1 \leq q \leq Q_{y d}$.

In the online stage, given a new parameter $\mu \in \mathcal{D}$ and associated optimal solutions $x_{N}^{*}$ and $\hat{x}_{M}^{*}$, we evaluate the residual correction terms from

$$
\begin{aligned}
r_{y}\left(\hat{y}_{M} ; \mu\right) & =\left\langle\mathcal{B} u_{N}, \hat{y}_{M}\right\rangle_{Y^{\prime}, Y}-a\left(y_{N}, \hat{y}_{M} ; \mu\right) \\
& =\left(B_{M} u_{N}\right)^{T} \underline{\hat{y}}_{M}-\sum_{q=1}^{Q_{a}} \Theta_{a}^{q}(\mu) \underline{y}_{N}^{T} A_{N, M}^{q} \underline{\hat{y}}_{M}
\end{aligned}
$$

and

$$
\begin{aligned}
r_{p}\left(\hat{p}_{M} ; \mu\right) & =\left(y_{d}(\mu), \hat{p}_{M}\right)_{\mathrm{L}^{2}(D)}-\left(y_{N}, \hat{p}_{M}\right)_{\mathrm{L}^{2}(D)}-a\left(\hat{p}_{M}, p_{N} ; \mu\right) \\
& =\sum_{q=1}^{Q_{y d}} \Theta_{y d}^{q}(\mu)\left(Y_{d, M}^{q}\right)^{T} \underline{\hat{p}}_{M}-\underline{y}_{N}^{T} D_{N, M} \underline{\hat{p}}_{M}-\sum_{q=1}^{Q_{a}} \Theta_{a}^{q}(\mu) \underline{\hat{p}}_{M}^{T} A_{M, N}^{q} \underline{p}_{N} .
\end{aligned}
$$

The computational cost to evaluate $\Delta_{N, M}^{\ell, *}(\mu)$ online - in addition to the cost for evaluating the primal state and adjoint residuals - is $\mathcal{O}\left(\left(Q_{a} N+m\right)^{2}+\left(Q_{a} N+N+Q_{y d}\right)^{2}+\left(Q_{a} M+m+1\right)^{2}+\left(Q_{a} M+M+1\right)^{2}+Q_{a} N M+Q_{y d} M\right)$ and thus independent of $\mathcal{N}$. 


\subsection{Greedy algorithm}

We generate the primal and dual reduced basis spaces using the Greedy sampling procedure summarized in Algorithm 1 (exemplarily shown only for one dual problem). Here, $\Xi_{\text {train }}^{\mathrm{pr}} \subset \mathcal{D}$ and $\Xi_{\text {train }}^{\text {du }} \subset \mathcal{D}$ are finite but suitably large parameter train samples for the primal and dual problem, respectively; $\mu_{\mathrm{pr}}^{1} \in \Xi_{\text {train }}^{\mathrm{pr}}$ and $\mu_{\mathrm{du}}^{1} \in \Xi_{\text {train }}^{\mathrm{du}}$ are the initial parameter values; and $\epsilon_{\mathrm{tol}, \min }>0$ is a prescribed desired error tolerance. Note that we expand the primal and dual reduced basis spaces in steps 8 and 9 with a snapshot of the corresponding state and the adjoint equation, i.e., we use "integrated" spaces as discussed previously.

We make two remarks: First, if one is interested in the "primal-only" approach described in Sections 4.1 and 4.2, all steps in Algorithm 1 associated to the generation of the dual space can be ignored. In this case we propose to use the (relative) cost functional error bound, $\Delta_{N}^{J, *}(\mu) / J_{N}^{*}(\mu)$, for the sampling procedure due to its superconvergence property. Second, if the primal-dual approach is used, we propose to use the (relative) primal-dual cost functional bound, $\Delta_{N, M}(\mu)=\Delta_{N, M}^{J, *}(\mu) / J_{N}^{*}(\mu)$, for sampling the primal problem and $\Delta_{M}(\mu)=$ $\max \left(\Delta_{M}^{\hat{y}, *}(\mu), \Delta_{M}^{\hat{p}, *}(\mu)\right)$, i.e., the dual ingredients of the cost functional bound, for sampling the dual problem. The spaces are generated simultaneously since $\Delta_{N, M}(\mu)$ depends on the primal and dual problem. This choice is also used in Section 5 for the numerical results.

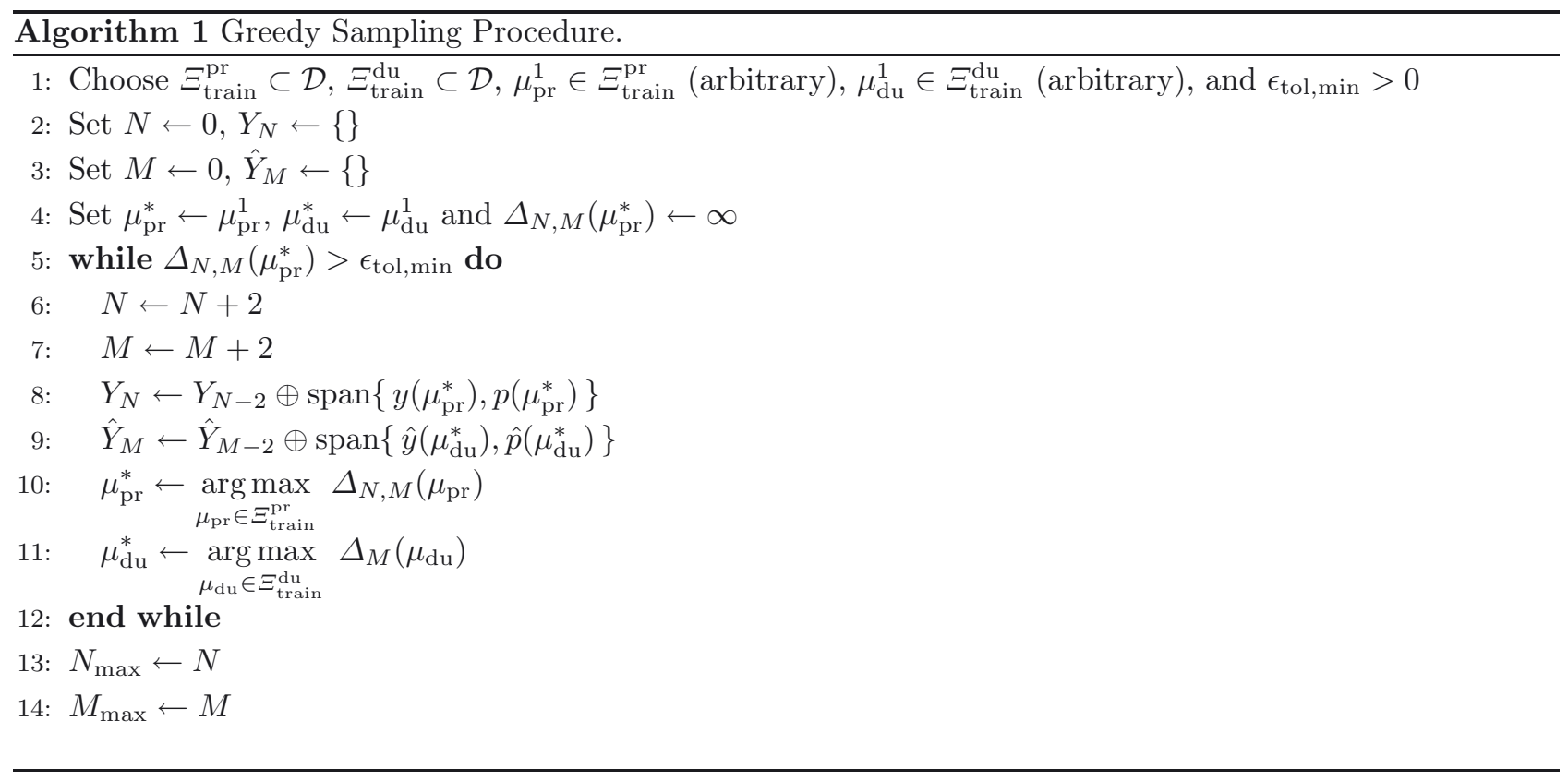

\section{Numerical RESUlts}

We consider a linear-quadratic optimal control problem governed by steady heat conduction in a twodimensional domain [22]. The spatial domain, a typical point of which is $x=\left(x_{1}, x_{2}\right)$, is given by $\Omega=$ $(0,7) \times(0,3)$ and is subdivided into the four subdomains $\Omega_{1}=((1,2) \times(0,1)) \cup((5,6) \times(0,1)), \Omega_{2}=(3,4) \times(0,1)$, $\Omega_{3}=(1,6) \times(2.5,3)$, and $\Omega_{4}=\Omega \backslash\left\{\Omega_{1} \cup \Omega_{2} \cup \Omega_{3}\right\}$. A sketch of the domain is shown in Figure 1 . The temperature satisfies Laplace's equation in $\Omega$ with continuity of temperature and heat flux across subdomain interfaces, i.e., on the interface of $\Omega_{1} \cup \Omega_{2}$ and $\Omega_{4}$ we have $\left.y\right|_{\Omega_{1} \cup \Omega_{2}}=\left.y\right|_{\Omega_{4}}$ and $\left.\frac{\partial}{\partial n} y\right|_{\Omega_{1} \cup \Omega_{2}}=\left.\mu_{1} \frac{\partial}{\partial n} y\right|_{\Omega_{4}}$. We impose zero Dirichlet conditions on the left and right boundaries and zero Neumann conditions on the bottom and top boundaries. The amount of heat supply in the heater domains $\Omega_{1}$ and $\Omega_{2}$ is regulated by the first and second 


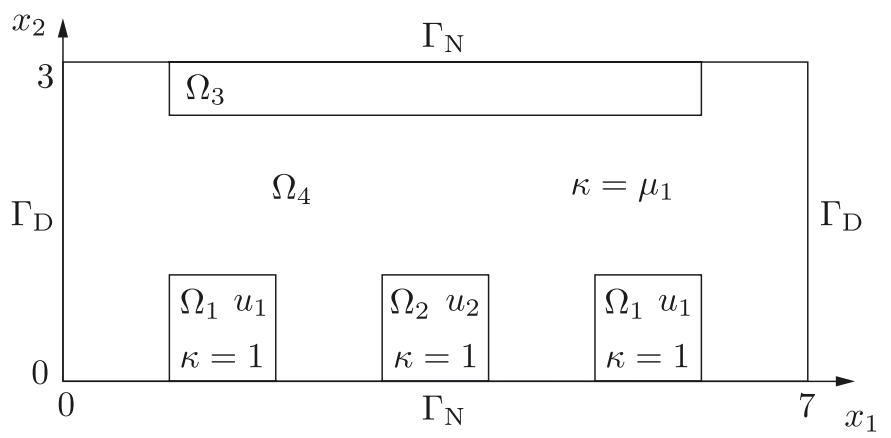

Figure 1. Domain $\Omega$ for the model problem.

component of the control $u=\left(u_{1}, u_{2}\right) \in \mathcal{U} \equiv \mathbb{R}^{2}$, respectively. The (reference) conductivity in the subdomain $\Omega_{1} \cup \Omega_{2}$ is set to unity. We consider the normalized conductivity $\kappa$ in the subdomain $\Omega_{3} \cup \Omega_{4}$ as our parameter $\mu_{1} \in[0.5,5]$.

The temperature $y(\mu) \in Y$ thus satisfies (2.8), where $Y \subset Y^{\mathrm{e}} \equiv\left\{v \in \mathrm{H}^{1}(\Omega):\left.v\right|_{\Gamma_{D}}=0\right\}$ is a linear finite element truth approximation subspace of dimension $\mathcal{N}=8479$ over a triangulation of $\Omega$. The bilinear and linear forms are given by $a(w, v ; \mu)=\mu_{1} \int_{\Omega_{3} \cup \Omega_{4}} \nabla w \nabla v \mathrm{~d} x+\int_{\Omega_{1} \cup \Omega_{2}} \nabla w \nabla v \mathrm{~d} x$ and $b_{i}(v)=\int_{\Omega_{i}} v \mathrm{~d} x$ for $i=1,2$, respectively. The bilinear form $a(\cdot, \cdot ; \mu)$ admits the affine representation $(2.3)$ with $\Theta_{a}^{1}(\mu)=\mu_{1}, \Theta_{a}^{2}(\mu)=1$ and $Q_{a}=2$. We also define the inner product $(w, v)_{Y}=\mu_{1}^{\text {ref }} \int_{\Omega_{3} \cup \Omega_{4}} \nabla w \nabla v \mathrm{~d} x+\int_{\Omega_{1} \cup \Omega_{2}} \nabla w \nabla v \mathrm{~d} x$ for $\mu_{1}^{\text {ref }}=\sqrt{2.5}$; we may hence choose $\alpha_{\mathrm{LB}}(\mu)=\min \left(\mu_{1} / \mu_{1}^{\text {ref }}, 1\right)$ in $(2.10)$.

We consider the quadratic cost-functional $J(y, u ; \mu)=\frac{1}{2}\left\|y-y_{d}(\mu)\right\|_{\mathrm{L}^{2}(D)}^{2}+\frac{\lambda}{2}\left\|u-u_{d}\right\|_{\mathcal{U}}^{2}$, with $u_{d}=(1,1)^{T}$ and $D=\Omega_{3}$. Here, the parameter-dependent desired state $y_{d}(\cdot ; \mu): D \rightarrow \mathbb{R}$ is given by

$$
y_{d}(x ; \mu)=\mu_{2}\left(x_{1}-3.5\right)^{2}+1,
$$

where the parameter $\mu_{2}$ varies from -0.1 to 0.1 . The desired state $y_{d}(\mu)$ clearly satisfies the affine parameter dependence (3.2) with $Q_{y d}=2: \Theta_{y d}^{1}(\mu)=\mu_{2}, \Theta_{y d}^{2}=1, y_{d}^{1}(x)=\left(x_{1}-3.5\right)^{2}$, and $y_{d}^{2}(x)=1$. Finally, we also assume that the regularization parameter $\lambda$ is allowed to vary in the range from 0.01 to 1 . The full parametrization of our problem is thus given by $\mu=\left(\mu_{1}, \mu_{2}, \mu_{3}=\lambda\right) \in \mathcal{D} \equiv[0.5,5] \times[-0.1,0.1] \times[0.01,1]$; we have $P=3$ parameters.

We first present results for the solution of the truth optimal control problem $(\mathrm{P})$ for different parameter combinations. In Figure 2 we plot the optimal temperature distribution and state the optimal control and associated cost functional value. We note that all parameters have a strong influence on the solution of the optimal control problem: the temperature and optimal control vary significantly and the variation in the cost functional is almost three orders of magnitude. The range in the cost functional is largely due to the second parameter value, $\mu_{2}$, which influences the desired temperature profile in $\Omega_{3}$ : positive values of $\mu_{2}$ correspond to a parabola facing up and negative values to a parabola facing down. The optimal control problem can achieve a good fit - and thus small tracking error - only for negative values of $\mu_{2}$ because of the Dirichlet boundary condition.

We construct the reduced basis space $Y_{N}$ according to the Greedy sampling procedure described in Section 4.5. To this end, we employ the train samples $\Xi_{\text {train }}^{\mathrm{pr}} \subset \mathcal{D}$ consisting of $n_{\mathrm{train}}^{\mathrm{pr}}=845$ parameter points over $\mathcal{D}$ for the primal and $\Xi_{\text {train }}^{\text {du }} \subset \hat{\mathcal{D}}$ of size $n_{\text {train }}^{\text {du }}=169$ for the dual problem. Here $\hat{\mathcal{D}}=[0.5,5] \times[0.01,1]$ is the parameter domain for the dual problem ${ }^{6}$. We sample the primal problem on the relative primal-dual cost functional error bound $\Delta_{N, M}^{J, *}(\mu) / J_{N}^{*}(\mu)$ and the dual problem on the maximum of the dual state and adjoint optimality error

\footnotetext{
${ }^{6}$ Note that the second parameter, $\mu_{2}$, does not enter the dual problem (4.24) and is thus also not included in the Greedy sampling procedure.
} 

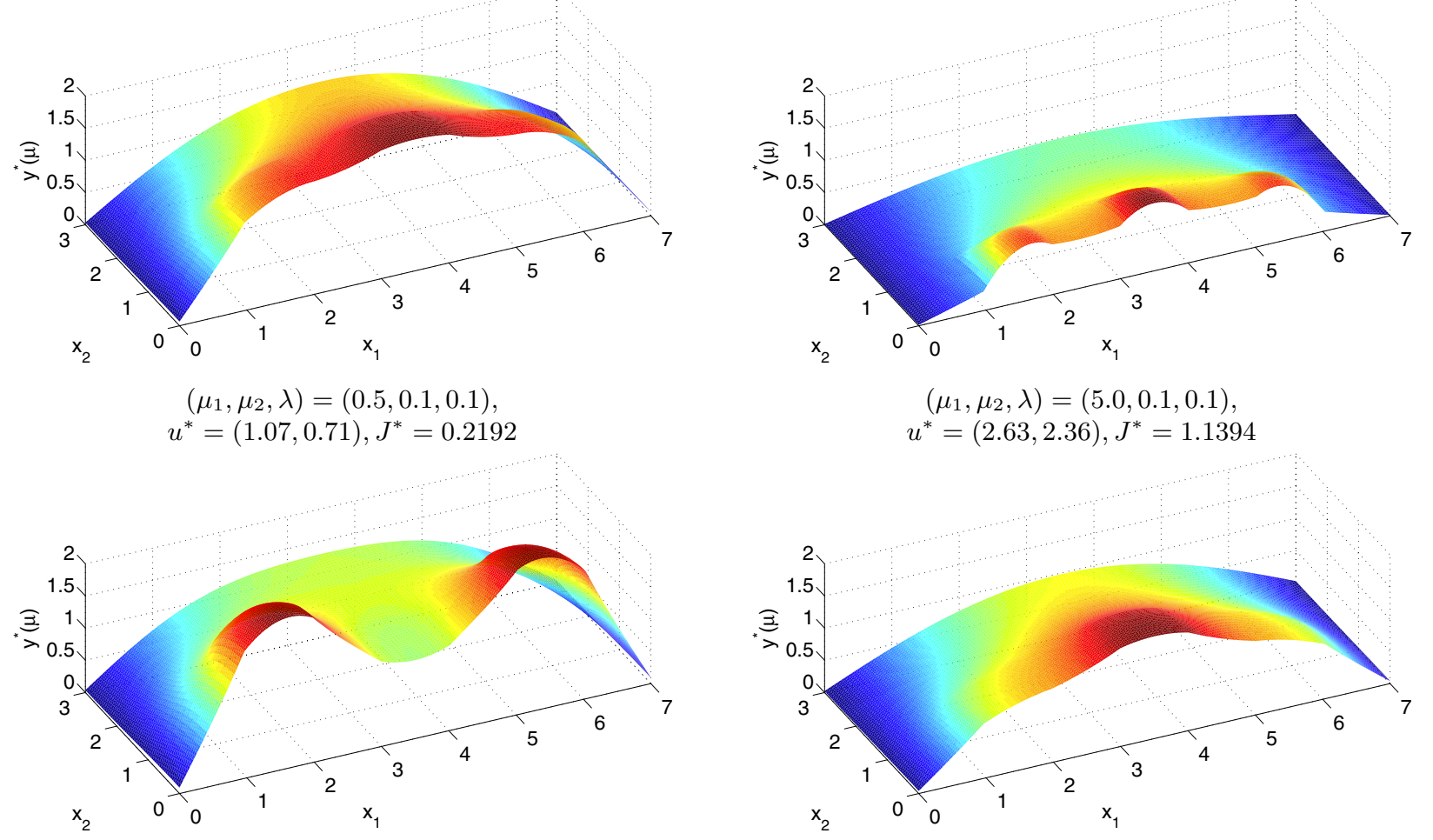

$$
\begin{gathered}
\left(\mu_{1}, \mu_{2}, \lambda\right)=(0.5,0.1,0.01), \\
u^{*}=(2.32,-0.88), J^{*}=0.1907
\end{gathered}
$$
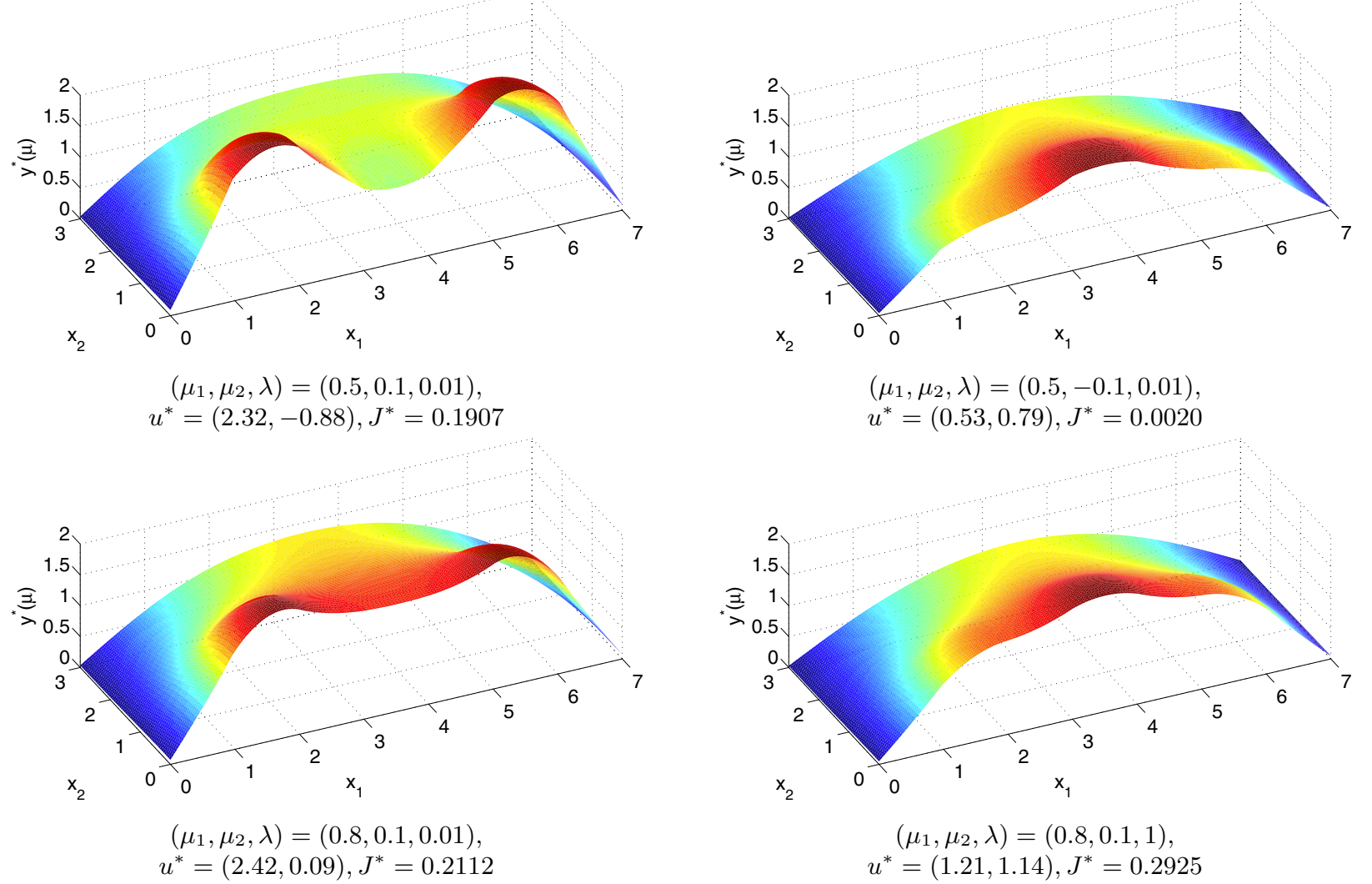

FiguRE 2. Optimal state $y^{*}(\mu)$, optimal control $u^{*}(\mu)$, and optimal cost functional value $J^{*}(\mu)$ for different representative parameter values.

bounds $\max \left(\Delta_{M}^{\hat{y}, *}(\mu), \Delta_{M}^{\hat{p}, *}(\mu)\right)$. The desired error tolerance is $\epsilon_{\text {tol,min }}=1 \mathrm{E}-10$ and the initial parameter values are $\mu_{\mathrm{pr}}^{1}=(0.5,-0.1,0.01)$ and $\mu_{\mathrm{du}}^{1}=(0.5,0.01)$. We also introduce a parameter test sample $\Xi_{\text {test }}$ of size $n_{\text {test }}=100$ with a log-random distribution in $\mu_{1}$ and $\lambda$ and a uniform-random distribution in $\mu_{2}$.

In Table 1 we present, as a function of $N$, the maximum relative predictability errors $\epsilon_{N, \max , \text { rel }}^{y}$ and $\epsilon_{N, \max , \mathrm{rel}}^{p}$, the maximum relative error bounds $\tilde{\Delta}_{N \text {,max,rel }}^{y}$ and $\tilde{\Delta}_{N, \text { max, rel }}^{p}$, and the average effectivities $\bar{\eta}_{N}^{y}$ and $\bar{\eta}_{N}^{p}$ for the state and adjoint equation, respectively. Here, $\epsilon_{N, \max , \text { rel }}^{y}$ is the maximum over $\Xi_{\text {test }}$ of $\left\|\tilde{e}^{y}(\mu)\right\|_{Y} /\left\|y\left(u_{N}^{*}(\mu)\right)\right\|_{Y}$, 
TABLE 1. State and adjoint predictability errors, error bounds, and effectivities as a function of $N$.

\begin{tabular}{l|ccc|ccc}
\hline \multicolumn{5}{c}{ State } \\
\hline$N$ & $\epsilon_{N, \text { max }, \mathrm{rel}}^{y}$ & $\tilde{\Delta}_{N, \text { max }, \mathrm{rel}}^{y}$ & $\bar{\eta}_{N}^{y}$ & $\epsilon_{N, \mathrm{max}, \mathrm{rel}}^{p}$ & $\tilde{\Delta}_{N, \text { max }, \mathrm{rel}}^{p}$ & $\bar{\eta}_{N}^{p}$ \\
\hline 2 & $6.74 \mathrm{E}-01$ & $9.16 \mathrm{E}-01$ & $1.20 \mathrm{E}+00$ & $1.01 \mathrm{E}+00$ & $4.38 \mathrm{E}+01$ & $1.57 \mathrm{E}+01$ \\
4 & $5.39 \mathrm{E}-01$ & $6.94 \mathrm{E}-01$ & $1.25 \mathrm{E}+00$ & $3.16 \mathrm{E}-01$ & $1.52 \mathrm{E}+01$ & $1.28 \mathrm{E}+01$ \\
8 & $4.72 \mathrm{E}-02$ & $5.22 \mathrm{E}-02$ & $1.43 \mathrm{E}+00$ & $1.01 \mathrm{E}-01$ & $1.33 \mathrm{E}+00$ & $9.48 \mathrm{E}+00$ \\
12 & $4.69 \mathrm{E}-03$ & $9.77 \mathrm{E}-03$ & $1.41 \mathrm{E}+00$ & $1.75 \mathrm{E}-02$ & $1.22 \mathrm{E}-01$ & $5.50 \mathrm{E}+00$ \\
16 & $1.61 \mathrm{E}-04$ & $1.66 \mathrm{E}-04$ & $1.43 \mathrm{E}+00$ & $7.56 \mathrm{E}-03$ & $7.93 \mathrm{E}-03$ & $3.12 \mathrm{E}+00$ \\
20 & $4.21 \mathrm{E}-06$ & $7.69 \mathrm{E}-06$ & $1.45 \mathrm{E}+00$ & $2.08 \mathrm{E}-04$ & $3.38 \mathrm{E}-04$ & $2.00 \mathrm{E}+00$ \\
24 & $1.92 \mathrm{E}-07$ & $2.72 \mathrm{E}-07$ & $1.50 \mathrm{E}+00$ & $6.09 \mathrm{E}-05$ & $6.41 \mathrm{E}-05$ & $1.60 \mathrm{E}+00$ \\
\hline
\end{tabular}

TABle 2. Control: error convergence, error bounds, and effectivities as a function of $N$.

\begin{tabular}{l|c|cc|cc}
\hline$N=M$ & $\epsilon_{N, \text { max }, \text { rel }}^{u, *}$ & $\Delta_{N, \text { max,rel }}^{u, *}$ & $\bar{\eta}_{N}^{u, *}$ & $\Delta_{N, M, \text { max }, \text { rel }}^{u, *}$ & $\bar{\eta}_{N, M}^{u, *}$ \\
\hline 2 & $6.64 \mathrm{E}-02$ & $3.23 \mathrm{E}+02$ & $8.68 \mathrm{E}+02$ & $5.53 \mathrm{E}+03$ & $2.98 \mathrm{E}+04$ \\
4 & $6.63 \mathrm{E}-02$ & $3.42 \mathrm{E}+02$ & $3.08 \mathrm{E}+03$ & $1.84 \mathrm{E}+02$ & $6.26 \mathrm{E}+03$ \\
8 & $3.12 \mathrm{E}-03$ & $1.08 \mathrm{E}+01$ & $3.72 \mathrm{E}+03$ & $6.95 \mathrm{E}+00$ & $1.16 \mathrm{E}+03$ \\
12 & $6.27 \mathrm{E}-05$ & $6.24 \mathrm{E}-01$ & $2.44 \mathrm{E}+04$ & $2.85 \mathrm{E}-02$ & $2.58 \mathrm{E}+03$ \\
16 & $2.15 \mathrm{E}-07$ & $7.74 \mathrm{E}-02$ & $4.45 \mathrm{E}+05$ & $4.95 \mathrm{E}-05$ & $3.37 \mathrm{E}+02$ \\
20 & $1.70 \mathrm{E}-09$ & $8.98 \mathrm{E}-04$ & $5.56 \mathrm{E}+06$ & $3.32 \mathrm{E}-08$ & $2.12 \mathrm{E}+02$ \\
24 & $1.80 \mathrm{E}-11$ & $1.57 \mathrm{E}-04$ & $4.14 \mathrm{E}+07$ & $4.13 \mathrm{E}-10$ & $1.04 \mathrm{E}+02$ \\
\hline
\end{tabular}

$\tilde{\Delta}_{N, \text { max }, \text { rel }}^{y}$ is the maximum over $\Xi_{\text {test }}$ of $\tilde{\Delta}_{N}^{y}(\mu) /\left\|y\left(u_{N}^{*}(\mu)\right)\right\|_{Y}$, and $\bar{\eta}_{N}^{y}$ is the average over $\Xi_{\text {test }}$ of $\tilde{\Delta}_{N}^{y}(\mu) / \tilde{e}^{y}(\mu)$. The quantities for the adjoint variable are defined analogously (normalized by $\left.\left\|p\left(y\left(u_{N}^{*}(\mu)\right)\right)\right\|_{Y}\right)$. We observe that the state and adjoint predictability errors and bounds are decreasing very rapidly with increasing dimension of the reduced basis space and that the error bounds are very sharp for all values of $N$. The slightly larger effectivity of the adjoint error bound for small values of $N$ is due to the fact that the state error bound, i.e., the second term in (4.6), dominates at this point. However, for higher values of $N$ the dual norm of the adjoint residual becomes the main contributor to $\tilde{\Delta}_{N}^{p}(\mu)$, which leads to a very sharp error bound.

Given the predictability error bounds we can evaluate the error bound for the optimal control. In Table 2 we thus present, as a function of $N(=M)$, the maximum relative control error $\epsilon_{N \text {,max,rel }}^{u, *}$ and the primalonly and primal-dual control error bounds $\Delta_{N, \text { max,rel }}^{u, *}$ and $\Delta_{N, M, \text { max,rel }}^{u, *}$ and associated average effectivities $\bar{\eta}_{N}^{u, *}$ and $\bar{\eta}_{N, M}^{u, *}$, respectively. Here, $\epsilon_{N, \text { max,rel }}^{u, *}$ is the maximum over $\Xi_{\text {test }}$ of $\left\|u^{*}(\mu)-u_{N}^{*}(\mu)\right\|_{\mathcal{U}} /\left\|u^{*}(\mu)\right\|_{\mathcal{U}} ; \Delta_{N, \text { max,rel }}^{u, *}$ and $\Delta_{N, M, \text { max,rel }}^{u, *}$ are the maxima over $\Xi_{\text {test }}$ of $\Delta_{N}^{u, *}(\mu) /\left\|u^{*}(\mu)\right\|_{\mathcal{U}}$ and $\Delta_{N, M}^{u, *}(\mu) /\left\|u^{*}(\mu)\right\|_{\mathcal{U}}$, respectively; and $\bar{\eta}_{N}^{u, *}$ and $\bar{\eta}_{N, M}^{u, *}$ are the averages over $\Xi_{\text {test }}$ of $\Delta_{N}^{u, *}(\mu) /\left\|u^{*}(\mu)-u_{N}^{*}(\mu)\right\|_{\mathcal{U}}$ and $\Delta_{N, M}^{u, *}(\mu) /\left\|u^{*}(\mu)-u_{N}^{*}(\mu)\right\|_{\mathcal{U}}$. We observe that the control error exhibits a superlinear convergence with respect to the state and adjoint predictability errors. The primal-only control error bound is not able to capture the error decay in the control and the mean effectivity thus deteriorates considerably as $N$ increases. In contrast, the primal-dual error bound is able to match the convergence behavior of the actual error and the effectivities even decrease slightly with increasing $N$. We recall that evaluation of the primal-dual control error bound requires the solution of two dual optimality systems in addition to the primal optimality system. Here, and for the rest of this section, we assume that the reduced basis dimensions of the two dual problems are equivalent.

We next turn to the optimality errors. In Table 3 we present, as a function of $N$, the maximum relative optimality errors $\epsilon_{N \text {,max,rel }}^{y, *}$ and $\epsilon_{N, \text { max,rel }}^{p, *}$, the maximum relative error bounds $\Delta_{N, \text { max,rel }}^{y, *}$ and $\Delta_{N, \text { max,rel }}^{p, *}$, and 
TABLE 3. State and adjoint optimality errors, error bounds, and effectivities as a function of $N$.

\begin{tabular}{l|ccc|ccc}
\hline & \multicolumn{3}{|c|}{ State } & \multicolumn{3}{c}{ Adjoint } \\
\hline$N$ & $\epsilon_{N, \text { max }, \text { rel }}^{y, *}$ & $\Delta_{N, \text { max,rel }}^{y, *}$ & $\bar{\eta}_{N}^{y, *}$ & $\epsilon_{N, \text { max }, \text { rel }}^{p, *}$ & $\Delta_{N, \text { max }, \text { rel }}^{p, *}$ & $\bar{\eta}_{N}^{p, *}$ \\
\hline 2 & $6.70 \mathrm{E}-01$ & $5.28 \mathrm{E}+02$ & $7.93 \mathrm{E}+01$ & $8.06 \mathrm{E}-01$ & $1.30 \mathrm{E}+04$ & $2.55 \mathrm{E}+03$ \\
4 & $5.36 \mathrm{E}-01$ & $5.60 \mathrm{E}+02$ & $8.44 \mathrm{E}+01$ & $3.17 \mathrm{E}-01$ & $1.15 \mathrm{E}+04$ & $3.18 \mathrm{E}+03$ \\
8 & $4.68 \mathrm{E}-02$ & $1.70 \mathrm{E}+01$ & $1.28 \mathrm{E}+02$ & $1.01 \mathrm{E}-01$ & $3.09 \mathrm{E}+02$ & $2.06 \mathrm{E}+03$ \\
12 & $4.69 \mathrm{E}-03$ & $1.02 \mathrm{E}+00$ & $1.85 \mathrm{E}+02$ & $1.75 \mathrm{E}-02$ & $1.86 \mathrm{E}+01$ & $6.16 \mathrm{E}+02$ \\
16 & $1.61 \mathrm{E}-04$ & $1.27 \mathrm{E}-01$ & $7.22 \mathrm{E}+02$ & $7.56 \mathrm{E}-03$ & $2.31 \mathrm{E}+00$ & $3.25 \mathrm{E}+02$ \\
20 & $4.21 \mathrm{E}-06$ & $1.31 \mathrm{E}-03$ & $2.10 \mathrm{E}+03$ & $2.08 \mathrm{E}-04$ & $6.90 \mathrm{E}-02$ & $1.46 \mathrm{E}+02$ \\
24 & $1.92 \mathrm{E}-07$ & $2.07 \mathrm{E}-04$ & $1.70 \mathrm{E}+04$ & $6.09 \mathrm{E}-05$ & $7.00 \mathrm{E}-03$ & $9.83 \mathrm{E}+01$ \\
\hline
\end{tabular}

TABLE 4. State and adjoint optimality errors, dual error bounds, and effectivities as a function of $N$.

\begin{tabular}{l|ccc|ccc}
\hline & \multicolumn{3}{|c|}{ State } & \multicolumn{3}{c}{ Adjoint } \\
\hline$N=M$ & $\epsilon_{N, \text { max }, \text { rel }}^{y, *}$ & $\Delta_{N, M, \text { max,rel }}^{y, *}$ & $\bar{\eta}_{N, M}^{y, *}$ & $\epsilon_{N, \text { max }, \mathrm{rel}}^{p, *}$ & $\Delta_{N, M, \text { max rel }}^{p, *}$ & $\bar{\eta}_{N, M}^{p, *}$ \\
\hline 2 & $6.70 \mathrm{E}-01$ & $9.05 \mathrm{E}+03$ & $2.59 \mathrm{E}+03$ & $8.06 \mathrm{E}-01$ & $6.69 \mathrm{E}+05$ & $9.21 \mathrm{E}+04$ \\
4 & $5.36 \mathrm{E}-01$ & $2.76 \mathrm{E}+02$ & $1.89 \mathrm{E}+02$ & $3.17 \mathrm{E}-01$ & $9.04 \mathrm{E}+03$ & $4.52 \mathrm{E}+03$ \\
8 & $4.68 \mathrm{E}-02$ & $8.01 \mathrm{E}+00$ & $3.75 \mathrm{E}+01$ & $1.01 \mathrm{E}-01$ & $1.74 \mathrm{E}+02$ & $6.01 \mathrm{E}+02$ \\
12 & $4.69 \mathrm{E}-03$ & $5.79 \mathrm{E}-02$ & $1.36 \mathrm{E}+01$ & $1.75 \mathrm{E}-02$ & $1.64 \mathrm{E}+00$ & $2.74 \mathrm{E}+01$ \\
16 & $1.61 \mathrm{E}-04$ & $2.22 \mathrm{E}-04$ & $2.02 \mathrm{E}+00$ & $7.56 \mathrm{E}-03$ & $8.00 \mathrm{E}-03$ & $3.44 \mathrm{E}+00$ \\
20 & $4.21 \mathrm{E}-06$ & $7.69 \mathrm{E}-06$ & $1.54 \mathrm{E}+00$ & $2.08 \mathrm{E}-04$ & $3.38 \mathrm{E}-04$ & $2.00 \mathrm{E}+00$ \\
24 & $1.92 \mathrm{E}-07$ & $2.72 \mathrm{E}-07$ & $1.56 \mathrm{E}+00$ & $6.09 \mathrm{E}-05$ & $6.41 \mathrm{E}-05$ & $1.60 \mathrm{E}+00$ \\
\hline
\end{tabular}

the average effectivities $\bar{\eta}_{N}^{y, *}$ and $\bar{\eta}_{N}^{p, *}$ for the state and adjoint variable, respectively. Here, $\epsilon_{N, \text { max.rel }}^{y, *}$ is the maximum over $\Xi_{\text {test }}$ of $\left\|e^{y, *}(\mu)\right\|_{Y} /\left\|y^{*}(\mu)\right\|_{Y}, \Delta_{N, \text { max,rel }}^{y, *}$ is the maximum over $\Xi_{\text {test }}$ of $\Delta_{N}^{y, *}(\mu) /\left\|y^{*}(\mu)\right\|_{Y}$, and $\bar{\eta}_{N}^{y, *}$ is the average over $\Xi_{\text {test }}$ of $\Delta_{N}^{y, *}(\mu) / e^{y, *}(\mu)$. The quantities for the adjoint variable are defined analogously. Again, we observe a rapid decay of both the state and adjoint optimality errors with increasing $N$. However, the effectivities of the state optimality error bounds increase with increasing $N$. This is caused by the control error bound, $\Delta_{N}^{u, *}(\mu)$ (see Tab. 2), which enters $\Delta_{N}^{y, *}(\mu)$ and limits the convergence. In contrast, the effectivities of the adjoint optimality error bounds decrease with increasing $N$. Although $\Delta_{N}^{u, *}(\mu)$ also enters the adjoint optimality bound $\Delta_{N}^{p, *}(\mu)$ indirectly through $\Delta_{N}^{y, *}(\mu)$, the bound $\Delta_{N}^{p, *}(\mu)$ is dominated by the dual norm of the adjoint residual for larger $N$ and thus the overestimation of the control has a reduced effect on $\Delta_{N}^{p, *}(\mu)$.

As pointed out in Section 4.3.3 we can employ the primal-dual control error bound to obtain improved optimality error bounds for the state and adjoint variable. We summarize the results in Table 4 . The quantities are defined analogously to Table 3, we simply replace the error bounds $\Delta_{N}^{y, *}(\mu)$ and $\Delta_{N}^{p, *}(\mu)$ by their respective counterparts $\Delta_{N, M}^{y, *}(\mu)$ and $\Delta_{N, M}^{p, *}(\mu)$. Except for small values of $N$ we now obtain very sharp bounds resulting in $\mathcal{O}(1-10)$ effectivities for the state and adjoint optimality errors.

We next consider the cost functional. In Table 5 we report, as a function of $N(=M)$, the maximum relative cost functional errors $\epsilon_{N, \text { max,rel }}^{J, *}$, the maximum relative error bounds $\Delta_{N, \text { max,rel }}^{J, *}$ and $\Delta_{N, M, \text { max,rel }}^{J, *}$, and the associated average effectivities $\bar{\eta}_{N}^{J, *}$ and $\bar{\eta}_{N, M}^{J, *}$. Here, $\epsilon_{N, \text { max }, \text { rel }}^{J, *}$ is the maximum over $\Xi_{\text {test }}$ of $\left|J^{*}(\mu)-J_{N}^{*}(\mu)\right| / J^{*}(\mu)$, $\Delta_{N, \text { max,rel }}^{J, *}$ and $\Delta_{N, M, \text { max,rel }}^{J, *}$ are the maxima over $\Xi_{\text {test }}$ of $\Delta_{N}^{J, *}(\mu) / J^{*}(\mu)$ and $\Delta_{N, M}^{J, *}(\mu) / J^{*}(\mu)$, respectively; and $\bar{\eta}_{N}^{J, *}$ and $\bar{\eta}_{N, M}^{J, *}$ are the averages over $\Xi_{\text {test }}$ of $\Delta_{N}^{J, *}(\mu) /\left|J^{*}(\mu)-J_{N}^{*}(\mu)\right|$ and $\Delta_{N, M}^{J, *}(\mu) /\left|J^{*}(\mu)-J_{N}^{*}(\mu)\right|$, respectively. 
TABLE 5. Cost functional: error convergence, error bounds, and effectivities as a function of $N$.

\begin{tabular}{l|l|lc|cc}
\hline$N=M$ & $\epsilon_{N, \text { max }, \text { rel }}^{J, *}$ & $\Delta_{N, \text { max }, \text { rel }}^{J, *}$ & $\bar{\eta}_{N}^{J, *}$ & $\Delta_{N, M, \text { max }, \text { rel }}^{J, *}$ & $\bar{\eta}_{N, M}^{J, *}$ \\
\hline 2 & $2.03 \mathrm{E}-01$ & $6.38 \mathrm{E}+04$ & $3.99 \mathrm{E}+04$ & $3.29 \mathrm{E}+06$ & $1.39 \mathrm{E}+06$ \\
4 & $1.74 \mathrm{E}-02$ & $2.19 \mathrm{E}+04$ & $1.80 \mathrm{E}+05$ & $1.61 \mathrm{E}+04$ & $2.59 \mathrm{E}+05$ \\
8 & $1.67 \mathrm{E}-03$ & $3.40 \mathrm{E}+01$ & $1.67 \mathrm{E}+04$ & $2.35 \mathrm{E}+01$ & $3.86 \mathrm{E}+03$ \\
12 & $5.53 \mathrm{E}-06$ & $6.11 \mathrm{E}-02$ & $9.92 \mathrm{E}+03$ & $8.18 \mathrm{E}-03$ & $1.40 \mathrm{E}+03$ \\
16 & $6.57 \mathrm{E}-08$ & $9.38 \mathrm{E}-04$ & $2.82 \mathrm{E}+04$ & $1.63 \mathrm{E}-06$ & $1.99 \mathrm{E}+02$ \\
20 & $2.32 \mathrm{E}-10$ & $5.35 \mathrm{E}-07$ & $2.33 \mathrm{E}+04$ & $1.24 \mathrm{E}-09$ & $6.91 \mathrm{E}+02$ \\
24 & $4.95 \mathrm{E}-12$ & $9.43 \mathrm{E}-09$ & $2.67 \mathrm{E}+04$ & $1.12 \mathrm{E}-11$ & $6.20 \mathrm{E}+01$ \\
\hline
\end{tabular}

TABLE 6. Output functional: error convergence, error bounds, and effectivities as a function of $N$.

\begin{tabular}{l|c|cc|cc}
\hline$N=M$ & $\epsilon_{N, \text { max }, \text { rel }}^{\ell, *}$ & $\Delta_{N, \text { max }, \text { rel }}^{\ell, *}$ & $\bar{\eta}_{N}^{\ell, *}$ & $\Delta_{N, M, \text { max }, \text { rel }}^{\ell, *}$ & $\bar{\eta}_{N, M}^{\ell, *}$ \\
\hline 2 & $1.28 \mathrm{E}-01$ & $1.12 \mathrm{E}+03$ & $6.52 \mathrm{E}+04$ & $1.77 \mathrm{E}+02$ & $3.33 \mathrm{E}+04$ \\
4 & $1.90 \mathrm{E}-02$ & $1.19 \mathrm{E}+03$ & $2.55 \mathrm{E}+06$ & $7.75 \mathrm{E}+01$ & $3.78 \mathrm{E}+05$ \\
8 & $4.77 \mathrm{E}-04$ & $3.61 \mathrm{E}+01$ & $3.89 \mathrm{E}+05$ & $7.34 \mathrm{E}-01$ & $4.73 \mathrm{E}+03$ \\
12 & $1.22 \mathrm{E}-06$ & $2.17 \mathrm{E}+00$ & $4.13 \mathrm{E}+06$ & $2.18 \mathrm{E}-03$ & $1.02 \mathrm{E}+04$ \\
16 & $1.68 \mathrm{E}-08$ & $2.69 \mathrm{E}-01$ & $6.10 \mathrm{E}+07$ & $1.39 \mathrm{E}-05$ & $9.37 \mathrm{E}+03$ \\
20 & $4.44 \mathrm{E}-11$ & $2.78 \mathrm{E}-03$ & $3.74 \mathrm{E}+09$ & $3.13 \mathrm{E}-08$ & $8.68 \mathrm{E}+03$ \\
24 & $1.16 \mathrm{E}-12$ & $3.74 \mathrm{E}-04$ & $6.44 \mathrm{E}+09$ & $5.52 \mathrm{E}-10$ & $1.88 \mathrm{E}+03$ \\
\hline
\end{tabular}

We note that - as opposed to the control - the error in the cost functional and the primal-only bound converge superlinearly with respect to the predictability and/or optimality errors and the effectivities thus do not deteriorate with increasing $N$. Unfortunately, the effectivities are fairly large for all values of $N$, i.e., we consistently overestimate the error in the cost functional. We therefore consider the improved primal-dual error bound (4.41): we observe that the primal-dual bound is indeed sharper for $N, M \geq 4$ and results in much smaller effectivities especially for larger values of $N, M$. We would also like to point out that, if the dual approach is used for the control error bound, the improvement in the cost functional bound is basically "for free," i.e., we do not need to solve an additional dual problem to obtain the sharper primal-dual error bound for the cost functional.

In Section 4.3.1 we derived the primal-dual bounds for a general linear output functional of the optimal solution. As a specific example, we consider the mean value of the temperature over the subdomain $\Omega_{3}$ corresponding to the linear output functional $\ell(z)=\ell_{y}(\phi)=\int_{\Omega_{3}} \phi \mathrm{d} x$ for $\phi=y^{*}$. We summarize the results in Table 6. Here, $\epsilon_{N, \text { max,rel }}^{\ell, *}, \Delta_{N, \text { max,rel }}^{\ell, *}, \bar{\eta}_{N}^{\ell, *}, \Delta_{N, M \text {,max,rel }}^{\ell, *}$, and $\bar{\eta}_{N, M}^{\ell, *}$ are defined analogously to the quantities for the cost functional, where the standard primal-only output error bound is given by $\Delta_{N}^{\ell, *}(\mu) \equiv\left\|\ell_{y}\right\|_{Y^{\prime}} \Delta_{N}^{y, *}(\mu)$. The error decay is very rapid and comparable to the control and cost functional error. The primal-only bound does not converge superlinearly and the effectivities thus deteriorate significantly as $N$ increases. The primaldual error bound, on the other hand, is able to capture the superlinear convergence of the output error: the corresponding effectivities decrease slightly with increasing $N$. The behavior is similar to the one we observed with the optimal control.

Next, we study the effect on the primal-dual error bounds if we choose different values for $N$ and $M$. In Figure 3 we plot the maximum relative control error $\epsilon_{N, \text { max,rel }}^{u, *}$ and primal-only error bound $\Delta_{N, \text { max,rel }}^{u, *}$ as a function of $N$ as well as the maximum relative primal-dual control error bound $\Delta_{N, M \text {,max,rel }}^{u, *}$ as function of $N$ for various values of $M$. The corresponding plots for the primal-dual cost functional and output bound are shown in Figures 4 and 5, respectively. We observe that the error bounds decrease for fixed $M$ as $N$ increases 


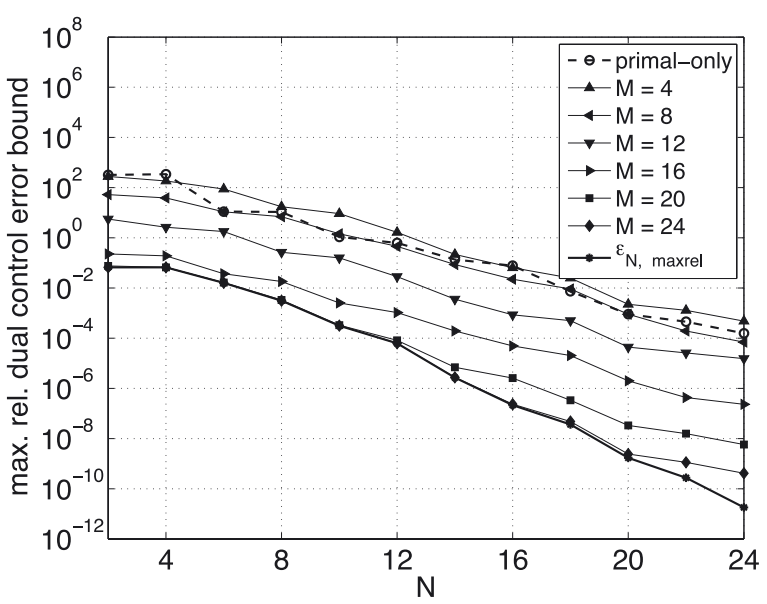

FiguRE 3. Maximum relative control error, primal-only error bound $\Delta_{N, \max , \mathrm{rel}}^{u, *}$, and primal-dual control error bound $\Delta_{N, M, \text { max,rel }}^{u, *}$ as a function of $N$ and $M$.

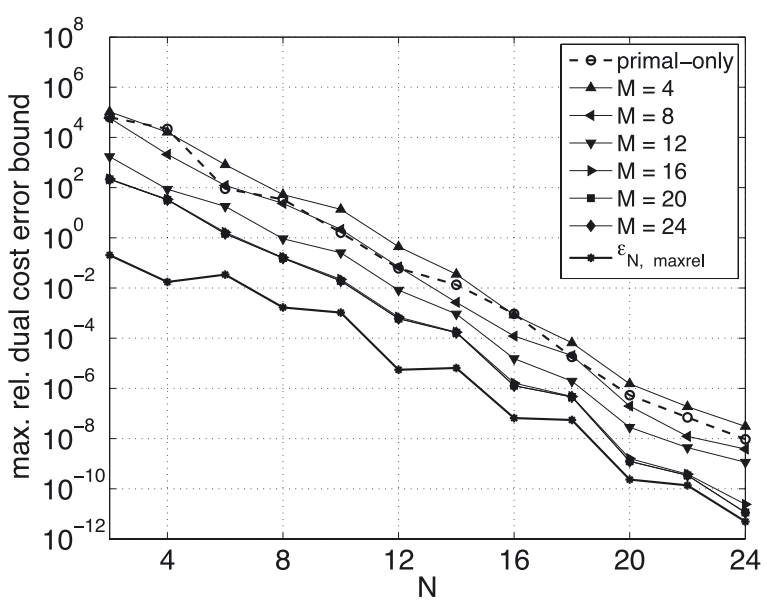

Figure 4. Maximum relative cost functional error, primal-only error bound $\Delta_{N, \text { max,rel }}^{J, *}$, and primaldual error bound $\Delta_{N, M, \text { max,rel }}^{J, *}$ as a function of $N$ and $M$.

TABLE 7. Control and cost functional: error convergence, error bounds, and effectivities as a function of $N$ and $M$.

\begin{tabular}{l|lllc|cccc}
\hline$N$ & $M$ & $\epsilon_{N, \text { max,rel }}^{u, *}$ & $\Delta_{N, M, \text { max,rel }}^{u, *}$ & $\bar{\eta}_{N, M}^{u, *}$ & $M$ & $\epsilon_{N, \text { max }, \text { rel }}^{J, *}$ & $\Delta_{N, M, \text { max,rel }}^{J, *}$ & $\bar{\eta}_{N, M}^{J, *}$ \\
\hline 2 & 20 & $6.64 \mathrm{E}-02$ & $7.62 \mathrm{E}-02$ & $1.03 \mathrm{E}+00$ & 16 & $2.03 \mathrm{E}-01$ & $2.22 \mathrm{E}+02$ & $4.12 \mathrm{E}+02$ \\
4 & 20 & $6.63 \mathrm{E}-02$ & $6.65 \mathrm{E}-02$ & $1.06 \mathrm{E}+00$ & 16 & $1.74 \mathrm{E}-02$ & $3.42 \mathrm{E}+01$ & $1.06 \mathrm{E}+03$ \\
8 & 20 & $3.12 \mathrm{E}-03$ & $3.35 \mathrm{E}-03$ & $1.12 \mathrm{E}+00$ & 16 & $1.67 \mathrm{E}-03$ & $1.65 \mathrm{E}-01$ & $9.33 \mathrm{E}+01$ \\
12 & 20 & $6.27 \mathrm{E}-05$ & $8.20 \mathrm{E}-05$ & $1.90 \mathrm{E}+00$ & 16 & $5.53 \mathrm{E}-06$ & $6.96 \mathrm{E}-04$ & $3.56 \mathrm{E}+02$ \\
16 & 24 & $2.15 \mathrm{E}-07$ & $2.34 \mathrm{E}-07$ & $2.59 \mathrm{E}+00$ & 16 & $6.57 \mathrm{E}-08$ & $1.63 \mathrm{E}-06$ & $1.99 \mathrm{E}+02$ \\
20 & 24 & $1.70 \mathrm{E}-09$ & $2.43 \mathrm{E}-09$ & $1.85 \mathrm{E}+01$ & 16 & $2.32 \mathrm{E}-10$ & $1.61 \mathrm{E}-09$ & $7.15 \mathrm{E}+02$ \\
24 & 24 & $1.80 \mathrm{E}-11$ & $4.13 \mathrm{E}-10$ & $1.04 \mathrm{E}+02$ & 20 & $4.95 \mathrm{E}-12$ & $1.14 \mathrm{E}-11$ & $6.32 \mathrm{E}+01$ \\
\hline
\end{tabular}

and vice versa. A specific desired accuracy of the bound can thus be achieved for different combinations of $N$ and $M$. Furthermore, we note from Figures 3-5 that the dual approach clearly improves the convergence rates of the control and output error bounds, whereas the convergence rate of the cost functional error bound is fairly insensitive to $M$. Considering the cost functional, a larger $M$ simply allows to "shift" the convergence curve of the error bound closer to the actual error resulting in a sharper bound.

We may thus select values of $N$ and $M$ so as to (say) minimize the computational cost involved to achieve a desired accuracy, or minimize the effectivity of the error bound. If the main interest is in sharp bounds and hence small effectivities, for example, we need to choose $M$ larger than $N$ for the problem at hand. We present results for different combinations of $N$ and $M$ in Table 7 for the control and cost and in Table 8 for the output. Here, we choose $N$ vs. $M$ based on Figures 3-5: for a given $N$ we select the smallest possible $M$ so as to minimize the error bound and thus the effectivity.

Finally, in Figure 6 we report the maximum relative primal-only error bound $\Delta_{N, \text { max,rel }}^{u, *}$ and primal-dual error bound $\Delta_{N, M, \text { max,rel }}^{u, *}$ with $N=M$ as a function of the estimated online computational cost, i.e., the theoretical operation count. The online cost for the primal-only approach includes the assembly and solution of 


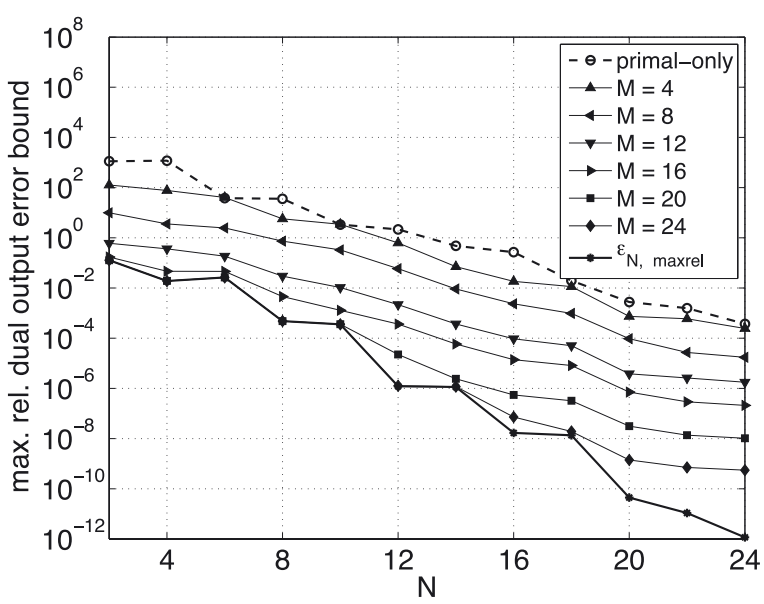

Figure 5. Maximum relative output error, primal-only error bound $\Delta_{N, \text { max }, \text { rel }}^{\ell, *}$, and primal-dual error bound $\Delta_{N, M, \text { max,rel }}^{\ell, *}$ as a function of $N$ and $M$.

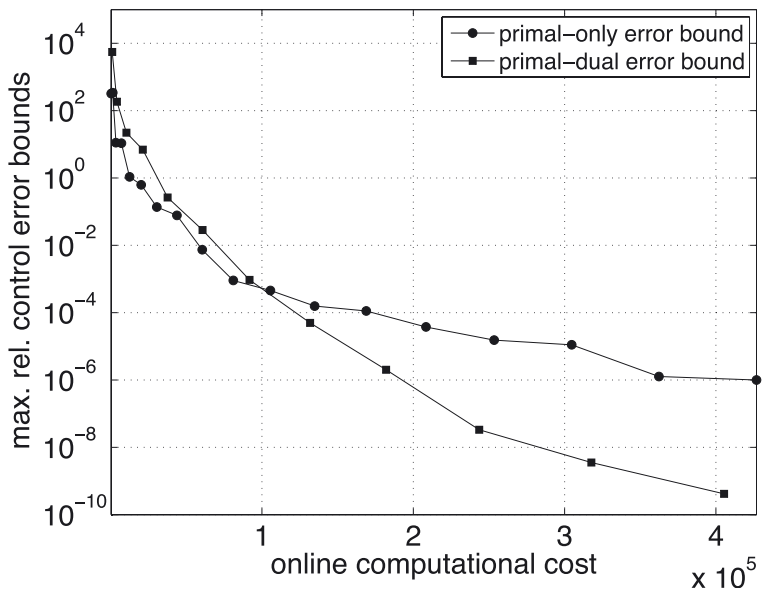

Figure 6. Maximum relative primal-only error bound $\Delta_{N, \text { max,rel }}^{u, *}$ and primal-dual error bound $\Delta_{N, M, \text { max,rel }}^{u, *}$ with $N=M$ as a function of the estimated online computational cost.

TABLE 8. Output functional: error convergence, error bounds, and effectivities as a function of $N$ and $M$.

\begin{tabular}{l|cccc}
\hline$N$ & $M$ & $\epsilon_{N, \text { max }, \text { rel }}^{\ell, *}$ & $\Delta_{N, M, \text { max }, \text { rel }}^{\ell, *}$ & $\bar{\eta}_{N, M}^{\ell, *}$ \\
\hline 2 & 20 & $1.28 \mathrm{E}-01$ & $1.29 \mathrm{E}-01$ & $1.19 \mathrm{E}+00$ \\
4 & 20 & $1.90 \mathrm{E}-02$ & $1.91 \mathrm{E}-02$ & $7.63 \mathrm{E}+00$ \\
8 & 20 & $4.77 \mathrm{E}-04$ & $4.81 \mathrm{E}-04$ & $1.81 \mathrm{E}+00$ \\
12 & 22 & $1.22 \mathrm{E}-06$ & $1.93 \mathrm{E}-06$ & $5.30 \mathrm{E}+00$ \\
16 & 24 & $1.68 \mathrm{E}-08$ & $7.22 \mathrm{E}-08$ & $2.02 \mathrm{E}+01$ \\
20 & 24 & $4.44 \mathrm{E}-11$ & $1.41 \mathrm{E}-09$ & $6.05 \mathrm{E}+02$ \\
24 & 24 & $1.16 \mathrm{E}-12$ & $5.52 \mathrm{E}-10$ & $1.88 \mathrm{E}+03$ \\
\hline
\end{tabular}

the primal reduced basis optimality system, the evaluation of the cost functional, and the computation of the primal-only control error bound $\Delta_{N}^{u, *}(\mu)$ as reported in Section 3.4 and 4.4.1. The online cost for the primaldual approach includes - in addition to the cost for the primal-only approach - the assembly and solution of the dual reduced basis optimality system (see Sect. 4.4.2) as well as the evaluation of the primal-dual control error bound $\Delta_{N, M}^{u, *}(\mu)$ (see Sect. 4.4.3). We observe that both approaches perform similarly in the beginning, i.e., for less accurate reduced basis approximations. The primal-dual approach, however, clearly outperforms the primal-only approach and thus results in considerable computational savings if one is interested in more accurate reduced basis approximations.

\section{ApPendix A. A POSTERIORI ERROR BOUNDS FOR DUAL OPTIMALITY SYSTEM}

We summarize the a posteriori error bounds for the dual optimality system. The results follow directly from the analysis of the primal optimality system in Sections 4.1 and 4.2. We start with the following 
Definition A.1. The residuals of the dual state equation, dual adjoint equation, and the dual optimality condition are defined as

$$
\begin{array}{lll}
\hat{r}_{\hat{p}}(\varphi ; \mu)=\ell_{p}(\varphi)+\left\langle\mathcal{B} \hat{u}_{M}^{*}, \varphi\right\rangle_{Y^{\prime}, Y}-a\left(\hat{p}_{M}^{*}, \varphi ; \mu\right), & \forall \varphi \in Y, & \forall \mu \in \mathcal{D}, \\
\hat{r}_{\hat{y}}(\phi ; \mu)=\ell_{y}(\phi)-\left(\phi, \hat{p}_{M}\right)_{\mathrm{L}^{2}(D)}-a\left(\phi, \hat{y}_{M}^{*} ; \mu\right), & \forall \phi \in Y, & \forall \mu \in \mathcal{D}, \\
\hat{r}_{\hat{u}}(\psi ; \mu)=\ell_{u}(\psi)-\left(\lambda \hat{u}_{M}^{*}-\mathcal{B}^{\star} \hat{y}_{M}^{*}, \psi\right)_{\mathcal{U}}, & \forall \psi \in \mathcal{U}, & \forall \mu \in \mathcal{D} .
\end{array}
$$

Again, since we do not employ a reduced basis for the low-dimensional control space $\mathcal{U}$ the residual of the optimality condition vanishes, i.e., we have $\hat{r}_{\hat{u}}(\psi ; \mu)=0, \forall \psi \in \mathcal{U}$. We next consider the dual predictability errors and the dual optimal control error.

Lemma A.2. The dual state predictability error, $\tilde{e}^{\hat{p}}=\hat{p}\left(\hat{u}_{M}^{*}\right)-\hat{p}_{M}^{*}\left(\hat{u}_{M}^{*}\right)$, is bounded by

$$
\left\|\tilde{e}^{\hat{p}}\right\|_{Y} \leq \tilde{\Delta}_{M}^{\hat{p}}(\mu) \equiv \frac{\left\|\hat{r}_{\hat{p}}(\cdot ; \mu)\right\|_{Y^{\prime}}}{\alpha_{\mathrm{LB}}(\mu)}, \quad \forall \mu \in \mathcal{D},
$$

where the dual adjoint residual $\hat{r}_{\hat{p}}(\varphi ; \mu)$ is defined in (A.1), $\hat{p}_{M}^{*}\left(\hat{u}_{M}^{*}\right)$ is the solution of $(4.28 \mathrm{~b})$, and $\hat{p}\left(\hat{u}_{M}^{*}\right)$ is the solution of the truth equation (4.26b) with control $\hat{u}_{M}^{*}$.

This is the standard a posteriori error bound for coercive elliptic PDEs, see Theorem 2.1.

Lemma A.3. The dual adjoint predictability error, $\tilde{e}^{\hat{y}}=\hat{y}\left(\hat{p}\left(\hat{u}_{M}^{*}\right)\right)-\hat{y}_{M}^{*}\left(\hat{p}_{M}^{*}\left(\hat{u}_{M}^{*}\right)\right)$, is bounded by

$$
\left\|\tilde{e}^{\hat{y}}\right\|_{Y} \leq \tilde{\Delta}_{M}^{\hat{y}}(\mu) \equiv \frac{1}{\alpha_{\mathrm{LB}}(\mu)}\left(\left\|\hat{r}_{\hat{y}}(\cdot ; \mu)\right\|_{Y^{\prime}}+C_{D}^{2} \tilde{\Delta}_{M}^{\hat{p}}(\mu)\right), \quad \forall \mu \in \mathcal{D},
$$

where the dual state residual $\hat{r}_{\hat{y}}(\phi ; \mu)$ is defined in (A.2), $C_{D} \equiv \sup _{v \in Y} \frac{\|v\|_{\mathrm{L}^{2}(D)}}{\|v\|_{Y}}, \hat{y}_{M}^{*}\left(\hat{p}_{M}^{*}\left(\hat{u}_{M}^{*}\right)\right)$ is the solution of (4.28a), and $\hat{y}\left(\hat{p}\left(\hat{u}_{M}^{*}\right)\right)$ is the solution of the truth equation (4.26a) with $\hat{p}\left(\hat{u}_{M}^{*}\right)$ instead of $\hat{p}^{*}\left(\hat{u}^{*}\right)$.

Proof. The desired result directly follows from the proof of Lemma 4.4 by replacing $\tilde{e}^{p}$ with $\tilde{e}^{\hat{y}}$ as well as $r_{p}(\varphi ; \mu)$ with $\hat{r}_{\hat{y}}(\phi ; \mu)$ and invoking Lemma A.2.

Proposition A.4. Let $\hat{u}^{*}$ and $\hat{u}_{M}^{*}$ be the optimal solutions of the dual truth and reduced basis optimality systems, respectively. Given $\tilde{\Delta}_{M}^{\hat{y}}(\mu)$ defined in (A.5), the error in the optimal control satisfies

$$
\left\|\hat{u}^{*}-\hat{u}_{M}^{*}\right\|_{\mathcal{U}} \leq \Delta_{M}^{\hat{u}, *}(\mu) \equiv \frac{1}{\lambda}\left(\sum_{i=1}^{m}\left\|b_{i}\right\|_{Y^{\prime}}^{2}\right)^{1 / 2} \tilde{\Delta}_{M}^{\hat{y}}(\mu), \quad \forall \mu \in \mathcal{D} .
$$

Proof. Following the proof of Theorem 4.11 in [31], one can show that

$$
\left\|\hat{u}^{*}-\hat{u}_{M}^{*}\right\|_{\mathcal{U}} \leq \frac{1}{\lambda}\left\|\lambda \hat{u}_{M}^{*}-\mathcal{B}^{\star} \hat{y}\left(\hat{p}\left(\hat{u}_{M}^{*}\right)\right)-\hat{\ell}_{u}\right\|_{\mathcal{U}}
$$

where $\hat{p}\left(\hat{u}_{M}^{*}\right)$ is the solution to the dual state equation (4.26b) with control $\hat{u}_{M}^{*}$ instead of $\hat{u}^{*}$ and $\hat{y}\left(\hat{p}\left(\hat{u}_{M}^{*}\right)\right)$ is the solution to the dual adjoint equation (4.26a) with $\hat{p}\left(\hat{u}_{M}^{*}\right)$ instead of $\hat{p}^{*}\left(\hat{u}^{*}\right)$. Furthermore $\hat{\ell}_{u} \in \mathcal{U}$ is the Riesz representation of $\ell_{u} \in \mathcal{U}^{\prime}$. The result then follows analogously to the proof of Proposition 4.5.

We finally turn to the dual optimality errors.

Lemma A.5. The dual state optimality error, $e^{\hat{p}, *}=\hat{p}^{*}\left(\hat{u}^{*}\right)-\hat{p}_{M}^{*}\left(\hat{u}_{M}^{*}\right)$, is bounded by

$$
\left\|e^{\hat{p}, *}\right\|_{Y} \leq \Delta_{M}^{\hat{p}, *}(\mu) \equiv \frac{1}{\alpha_{\mathrm{LB}}(\mu)}\left(\left\|\hat{r}_{\hat{p}}(\cdot ; \mu)\right\|_{Y^{\prime}}+\left(\sum_{i=1}^{m}\left\|b_{i}\right\|_{Y^{\prime}}^{2}\right)^{\frac{1}{2}} \Delta_{M}^{\hat{u}, *}(\mu)\right), \quad \forall \mu \in \mathcal{D} .
$$


Proof. The desired result directly follows from the proof of Lemma 4.6 by replacing $e^{y, *}$ with $e^{\hat{p}, *}$ as well as $r_{y}(\phi ; \mu)$ with $\hat{r}_{\hat{p}}(\varphi ; \mu)$ and invoking Proposition A.4.

Lemma A.6. The dual adjoint optimality error, $e^{\hat{y}, *}=\hat{y}^{*}\left(\hat{p}^{*}\left(\hat{u}^{*}\right)\right)-\hat{y}_{M}^{*}\left(\hat{p}_{M}^{*}\left(\hat{u}_{M}^{*}\right)\right)$, is bounded by

$$
\left\|e^{\hat{y}, *}\right\|_{Y} \leq \Delta_{M}^{\hat{y}, *}(\mu) \equiv \frac{1}{\alpha_{\mathrm{LB}}(\mu)}\left(\left\|\hat{r}_{\hat{y}}(\cdot ; \mu)\right\|_{Y^{\prime}}+C_{D}^{2} \Delta_{M}^{\hat{p}, *}(\mu)\right), \quad \forall \mu \in \mathcal{D} .
$$

Proof. The desired result directly follows from the proof of Lemma 4.7 by replacing $e^{p, *}$ with $e^{\hat{y}, *}$ as well as $r_{p}(\varphi ; \mu)$ with $\hat{r}_{\hat{y}}(\phi ; \mu)$ and invoking Lemma A.5.

\section{REFERENCES}

[1] J.A. Atwell and B.B. King, Proper orthogonal decomposition for reduced basis feedback controllers for parabolic equations. Math. Comput. Model. 33 (2001) 1-19.

[2] R. Becker, H. Kapp and R. Rannacher, Adaptive finite element methods for optimal control of partial differential equations: Basic concept. SIAM J. Control Optim. 39 (2000) 113-132.

[3] R. Becker and R. Rannacher, Weighted a posteriori error control in FE methods, in Proc. of ENUMATH-97. World Scientific Publishing (1998) 621-637.

[4] L. Dedè, Reduced basis method and a posteriori error estimation for parametrized linear-quadratic optimal control problems. SIAM J. Sci. Comput. 32 (2010) 997-1019.

[5] L. Dedè, Reduced basis method for parametrized elliptic advection-reaction problems. J. Comput. Math. 28 (2010) $122-148$.

[6] L. Dedè, Reduced basis method and error estimation for parametrized optimal control problems with control constraints. $J$. Sci. Comput. 50 (2012) 287-305.

[7] A.-L. Gerner and K. Veroy, Certified reduced basis methods for parametrized saddle point problems. Accepted in SIAM J. Sci. Comput. (2012).

[8] M.A. Grepl and M. Kärcher, Reduced basis a posteriori error bounds for parametrized linear-quadratic elliptic optimal control problems. C. R. Math. 349 (2011) 873-877.

[9] M.A. Grepl, Y. Maday, N.C. Nguyen and A.T. Patera, Efficient reduced-basis treatment of nonaffine and nonlinear partial differential equations. ESAIM: M2AN 41 (2007) 575-605.

[10] M.A. Grepl and A.T. Patera, A posteriori error bounds for reduced-basis approximations of parametrized parabolic partial differential equations. ESAIM: M2AN 39 (2005) 157-181.

[11] D.B.P. Huynh, G. Rozza, S. Sen and A.T. Patera, A successive constraint linear optimization method for lower bounds of parametric coercivity and inf-sup stability constants. C. R. Math. 345 (2007) 473-478.

[12] K. Ito and K. Kunisch, Reduced-order optimal control based on approximate inertial manifolds for nonlinear dynamical systems. SIAM J. Numer. Anal. 46 (2008) 2867-2891.

[13] K. Ito and S.S. Ravindran, A reduced basis method for control problems governed by pdes, in Control and Estimation of Distributed Parameter Systems, vol. 126 of Internat. Series Numer. Math., edited by W. Desch, F. Kappel and K. Kunisch. Birkhäuser Basel (1998) 153-168.

[14] K. Ito and S.S. Ravindran, A reduced-order method for simulation and control of fluid flows. J. Comput. Phys. 143 (1998) $403-425$.

[15] K. Ito and S.S. Ravindran, A reduced basis method for optimal control of unsteady viscous flows. Int. J. Comput. Fluid Dyn. 15 (2001) 97-113.

[16] M. Kärcher, The reduced-basis method for parametrized linear-quadratic elliptic optimal control problems, Master's thesis. Technische Universität München (2011).

[17] K. Kunisch and S. Volkwein, Control of the Burgers equation by a reduced-order approach using proper orthogonal decomposition. J. Optim. Theory Appl. 102 (1999) 345-371.

[18] K. Kunisch, S. Volkwein and L. Xie, HJB-POD based feedback design for the optimal control of evolution problems. SIAM J. Appl. Dyn. System 3 (2004) 701-722.

[19] J.L. Lions, Optimal Control of Systems Governed by Partial Differential Equations. Springer (1971).

[20] L. Machiels, Y. Maday, I.B. Oliveira, A.T. Patera and D.V. Rovas, Output bounds for reduced-basis approximations of symmetric positive definite eigenvalue problems. C. R. Math. 331 (2000) 153-158.

[21] F. Negri, Reduced basis method for parametrized optimal control problems governed by PDEs, Master's thesis. Politecnico di Milano (2011).

[22] I.B. Oliveira, A "HUM" Conjugate Gradient Algorithm for Constrained Nonlinear Optimal Control: Terminal and Regulator Problems, Ph.D. thesis. Massachusetts Institute of Technology (2002).

[23] M. Paraschivoiu, J. Peraire and A.T. Patera, A posteriori finite element bounds for linear-functional outputs of elliptic partial differential equations, Symposium on Advances in Computational Mechanics. Comput. Methods Appl. Mechanics Engrg. 150 (1997) 289-312. 
[24] N.A. Pierce and M.B. Giles, Adjoint recovery of superconvergent functionals from pde approximations. SIAM Review 42 (2000) 247-264.

[25] C. Prud'homme, D.V. Rovas, K. Veroy, L. Machiels, Y. Maday, A.T. Patera and G. Turinici, Reliable real-time solution of parametrized partial differential equations: Reduced-basis output bound methods. J. Fluids Engrg. 124 (2002) 70-80.

[26] A. Quarteroni, T. Lassila, A. Manzoni and G. Rozza, Boundary control and shape optimization for the robust design of bypass anastomoses under uncertainty. ESAIM: M2AN 47 (2013) 1107-1131.

[27] A. Quarteroni, G. Rozza, L. Dedè and A. Quaini, Numerical approximation of a control problem for advection-diffusion processes, System Modeling and Optimization, in vol. 199 of IFIP International Federation for Information Processing. Edited by F. Ceragioli, A. Dontchev, H. Futura, K. Marti and L. Pandolfi. Springer (2006) 261-273.

[28] A.M. Quarteroni and A. Valli, Numerical Approximation of Partial Differential Equations, vol. 23 of Springer Series in Comput. Math. Springer (2008).

[29] G. Rozza, D.B.P. Huynh and A.T. Patera, Reduced basis approximation and a posteriori error estimation for affinely parametrized elliptic coercive partial differential equations. Arch. Comput. Methods Engrg. 15 (2008) 229-275.

[30] T. Tonn, K. Urban and S. Volkwein, Comparison of the reduced-basis and pod a posteriori error estimators for an elliptic linear-quadratic optimal control problem. Math. Comput. Modell. Dyn. Syst. 17 (2011) 355-369.

[31] F. Tröltzsch and S. Volkwein, POD a posteriori error estimates for linear-quadratic optimal control problems. Comput. Optim. Appl. 44 (2009) 83-115.

[32] K. Veroy and A.T. Patera, Certifed real-time solution of the parametrized steady incompressible Navier-Stokes equations: rigorous reduced-basis a posteriori error bounds. Intern. J. Numer. Methods Fluids 47 (2005) 773-788.

[33] K. Veroy, C. Prud'homme, D.V. Rovas and A.T. Patera, A posteriori error bounds for reduced-basis approximation of parametrized noncoercive and nonlinear elliptic partial differential equations, in Proc. of the 16th AIAA Computational Fluid Dynamics Conference. AIAA Paper (2003) 2003-3847.

[34] K. Veroy, D.V. Rovas and A.T. Patera, A posteriori error estimation for reduced-basis approximation of parametrized elliptic coercive partial differential equations: "convex inverse" bound conditioners. Special volume: A tribute to J.L. Lions. ESAIM: COCV 8 (2002) 1007-1028.

[35] G. Vossen and S. Volkwein, Model reduction techniques with a posteriori error analysis for linear-quadratic optimal control problems, in vol. 298 of Konstanzer Schriften in Mathematik. Universität Konstanz (2012). 